SUNY-NTG-00/11

\title{
QCD-like Theories at Finite Baryon Density
}

\author{
J.B. Kogut ${ }^{1}$, M.A. Stephanov ${ }^{2}$, D. Toublan ${ }^{3}$, J.J.M. Verbaarschot ${ }^{3}$ and A. Zhitnitsky ${ }^{4}$ \\ ${ }^{1}$ Loomis Laboratory of Physics, University of Illinois, Urbana-Champaign, IL 61801, USA \\ ${ }^{2}$ Department of Physics, University of Illinois, Chicago, IL 60607-7059, USA \\ and \\ RIKEN-BNL Research Center, Brookhaven National Laboratory, Upton, NY 11973, USA \\ ${ }^{3}$ Department of Physics and Astronomy, SUNY, Stony Brook, New York 11794, USA \\ ${ }^{4}$ Department of Physics and Astronomy, University of British Columbia, Vancouver, BC \\ V6T 1Z1, Canada
}

\begin{abstract}
We study QCD-like theories with pseudoreal fermions at finite baryon density. Such theories include two-color QCD with quarks in the fundamental representation of the color group as well as any-color QCD with quarks in the adjoint color representation. In all such theories the lightest baryons are diquarks. At zero chemical potential $\mu$ they are, together with the pseudoscalar mesons, the Goldstone modes of a spontaneously broken enlarged chiral symmetry group. Using symmetry principles, we derive the low-energy effective Lagrangian for these particles. We find that a second order phase transition occurs at a value of $\mu$ equal to half the mass of the Goldstone modes. For values of $\mu$ beyond this point the scalar diquarks Bose condense and the diquark condensate is nonzero. We calculate the dependence of the chiral condensate, the diquark condensate, the baryon charge density, and the masses of the diquark and pseudoscalar excitations on $\mu$ at finite bare quark mass and scalar diquark source. The relevance of our results to lattice QCD calculations and to real three-color QCD at finite baryon density is discussed.
\end{abstract}

PACS: 11.30.Rd, 12.39.Fe, 12.38.Lg, 71.30.+h

Keywords: QCD partition function; Finite Baryon Density; QCD with two Colors; Adjoint QCD; QCD Dirac operator; Lattice QCD; Low-energy effective theory; Chiral Perturbation Theory 


\section{Introduction}

The study of strong interactions at finite baryon density has a long history. Phenomenological knowledge of nuclear forces allows one to obtain a good understanding of equilibrium nuclear matter which is relatively dilute. However, these results cannot be simply extended to higher densities relevant to neutron stars, supernova explosions and relativistic heavy ion collisions, where the microscopic degrees of freedom of QCD, quarks and gluons, become important.

Understanding QCD at finite density has been a tremendous challenge [1, 2]. Unlike finite temperature QCD, where significant progress has been achieved using lattice Monte Carlo simulations, QCD at finite baryon density is not amenable to such a numerical approach. The primary reason is that the determinant of the Euclidean QCD Dirac operator is not real at finite baryon chemical potential, $\mu$.

Recent progress has been achieved analytically by studying QCD at infinite density and by studying models of the Nambu-Jona-Lasinio type as well as the instanton liquid model [3]. It was found that the QCD vacuum at sufficiently high baryon density could become a color superconductor [3]. In other words, due to the attraction in the color anti-triplet isosinglet channel the quark Fermi surface becomes unstable towards the formation of a condensate of diquark pairs.

No first principle lattice calculation methods exist at this moment to study the phenomenon of color superconductivity. However, the mechanisms which lead to the formation of diquark condensates (gluon exchange or instanton induced attraction) can be investigated for QCD with two colors. This presents a tremendous advantage. QCD with two colors can be studied numerically on the lattice [4, 5] — the determinant of the Dirac operator is real. Another class of QCD-like theories with diquark condensation that can be studied on the lattice at nonzero chemical potential is QCD with any number of colors and of quarks in the adjoint color representation. Clearly, the physics of both types of theories is different from that of the three-color QCD, but the differences are easy to understand and classify. We hope that understanding the behavior of such theories at finite baryon number density will provide us with an additional insight into the phenomenon of diquark condensation in three-color QCD. In addition, numerical simulations of both QCD with two colors and QCD with adjoint quarks are now being pursued by several groups [5], 6].

The third class of theories which can be studied in the same way is QCD with the phase of the quark determinant quenched. In other words, for each quark such theory contains a conjugate quark, with opposite baryon charge. This leads to the appearance of colorless diquark states - baryonic pions. The zero-flavor limit $N_{f} \rightarrow 0$ of such a theory is the quenched approximation of QCD, as has been demonstrated analytically in [0] using a random matrix theory at nonzero $\mu$.

The unifying property of all such theories is the pseudo-reality of the quark representations, which manifests itself in the fact that the determinant of the Dirac operator is real. Another unifying property (which is ultimately related to this pseudo-reality) is the fact that diquarks can make color singlets - these are the baryons of such theories. The BoseEinstein condensation of diquarks, with nonzero baryon charge, may be viewed as baryon charge superconductivity (rather than color superconductivity as in three-color QCD).

In this paper we construct the low-energy chiral Lagrangian describing mesons and 
baryons (diquarks) at finite baryon density for two-color QCD and QCD with adjoint quarks. As was pointed out in [8] the $\mu$-dependence of this Lagrangian describing mesons and baryons can be fixed by global and certain local flavor symmetries. Within the domain of validity of this Lagrangian we study both its vacuum properties and the mass spectrum of the Goldstone modes. Our goal is twofold: (i) to understand and describe quantitatively the physics associated with diquark condensation; (ii) to provide lattice theorists with the qualitative and quantitative predictions aiding their data analysis.

This paper is organized as follows. In section 2 we give an overview of the different patterns of chiral symmetry breaking at $\mu=0$ for three-color QCD, two-color QCD and for QCD with adjoint quarks. We relate these patterns to the antiunitary symmetries of the Dirac operator, which provide us with a convenient classification in terms of the Dyson index $\beta$. Section 3 reviews the global symmetries of the theories, with the emphasis on the enlarged $S U\left(2 N_{f}\right)$ symmetry. The spontaneous breaking of this symmetry by the chiral condensate is the subject of section 1 , where we identify the Goldstone excitations. The effective Lagrangian at nonzero bare quark mass $m$ is introduced in section 5. In section 6 we review the introduction of the chemical potential into the effective Lagrangian by means of a local gauge principle [8, 9]. The vacuum alignment dictated by the effective Lagrangian is analyzed in sections 7 and 8 . In section 9 we expand the static part of Lagrangian around the minimum and in section 10 we determine the masses of the excitations as functions of $\mu$ and $m$ from the pole of their respective propagators. In section 11 we introduce the diquark source $j$ and find its effect on the vacuum and the mass spectrum. In section 12 we present the dependence of the vacuum condensates and the baryon number density on $\mu, m$ and $j$. Finally, in section 13, we rederive the equation of state of the dilute Bose gas of diquarks with repulsion and show that it exactly matches the equation of state obtained using our mean field analysis of the previous sections. Concluding remarks and discussion are presented in section 14.

\section{Overview and classification}

At zero chemical potential the spontaneous breaking of the chiral symmetry is believed to be an essential low-energy property of three color QCD with fundamental fermions. In the limit of massless quarks, the QCD Lagrangian is invariant under $U_{L}\left(N_{f}\right) \times U_{R}\left(N_{f}\right)$ transformations, but the ground state is not. The analysis of the hadron spectrum and numerical simulations on the lattice strongly support this assertion [10, 11]. The order parameter of the spontaneous breaking of chiral symmetry is the chiral quark-antiquark condensate. In the case of two-color QCD with fundamental fermions and in the case of any-color QCD with adjoint fermions the symmetry of the Lagrangian is enlarged from

$U_{L}\left(N_{f}\right) \times U_{R}\left(N_{f}\right)$ to $U\left(2 N_{f}\right)$. In the case of $N_{c}=2$, this symmetry is sometimes referred to as the Pauli-Gürsey symmetry [12]. Also in these cases there is strong evidence both from lattice simulations [13, 14 and from arguments based on supersymmetry [15 that chiral symmetry is broken by a nonzero vacuum expectation value of the chiral condensate.

The pattern of chiral symmetry breaking is determined by two ideas. The Vafa-Witten theorem [16] which tells us that vector symmetries cannot be spontaneously broken, and the idea of maximum breaking of the axial symmetry [17, 18]. For each of the three classes 
of theories chiral symmetry is broken spontaneously according to different patterns 『18, 17, 19]. The axial $\mathrm{U}(1)_{A}$ subgroup of the global symmetry is broken explicitly by the axial anomaly in all three cases. The vector-like $U(1)_{B}$ symmetry, corresponding to baryon charge conservation, is intact. The remaining symmetries in QCD with three or more colors with fundamental fermions are broken according to $S U_{R}\left(N_{f}\right) \times S U_{L}\left(N_{f}\right) \rightarrow S U_{V}\left(N_{f}\right)$. For twocolor QCD with fundamental fermions the symmetry is broken according to $S U\left(2 N_{f}\right) \rightarrow$ $S p\left(2 N_{f}\right)$, whereas for any-color QCD with adjoint fermions the pattern of symmetry breaking is given by $S U\left(2 N_{f}\right) \rightarrow O\left(2 N_{f}\right)$.

We can classify these above three cases by the Dyson index, $\beta$, of the Dirac operator with a value of $\beta=2, \beta=1$ and $\beta=4$, respectively. The value of $\beta$ is given by the number of independent degrees of freedom per matrix element and is determined by the antiunitary symmetries of the Dirac operator. It is a concept that originated in Random Matrix Theory 20, 21, 22], and is important for the Cartan classification of symmetric spaces [23].

In the case of two-color QCD the pseudo-real nature of $S U(2)_{\text {color }}$ [18, 17, 19, 24] can be expressed as the antiunitary symmetry of the Dirac operator $\mathcal{D}=\gamma_{\nu} D_{\nu}+m$,

$$
\left[\mathcal{D}, \tau_{2} C \gamma_{5} K\right]=0 \quad \text { or } \quad \mathcal{D} \tau_{2} C \gamma_{5}=\tau_{2} C \gamma_{5} \mathcal{D}^{*}, \quad(\beta=1)
$$

where $\tau_{2}$ is the color symmetry generator, $C$ is the Dirac charge conjugation matrix and $K$ is the complex conjugation operator. Since $\left(\tau_{2} C K\right)^{2}=1$ it is always possible to find a basis in which the Dirac operator becomes real which gives $\beta=1$.

The symmetry (11) persists even at $\mu \neq 0$ [25]. The reality of the Dirac determinant and the feasibility of lattice Monte Carlo simulations is the consequence of (11). This property also allows us to use QCD inequalities at finite $\mu$ to show that condensation can only occur in the scalar diquark channel [8].

In the case of QCD with adjoint quarks the antiunitary symmetry of the Dirac operator is

$$
\left[\mathcal{D}, C \gamma_{5} K\right]=0 \quad \text { or } \quad \mathcal{D} C \gamma_{5}=C \gamma_{5} \mathcal{D}^{*} \quad(\beta=4)
$$

Since $(C K)^{2}=-1$, it is always possible to find a basis in which the Dirac operator can be organized into selfdual (pseudoreal) quaternions. The value of the Dyson index is thus $\beta=4$.

In both above cases, $\beta=1$ and $\beta=4$, the antiunitary symmetry leads to enlargement of the global symmetry to $U\left(2 N_{f}\right)$ (at $\mu=0$ ).

There is no antiunitary symmetry in the case of QCD with three or more colors with fundamental quarks. The Dirac operator is a complex matrix, thus $\beta=2$.

In this paper we shall study two classes of theories: $\beta=1$ and $\beta=4$, at finite chemical potential $\mu$. The main starting point of our analysis is the observation of the fact that in these theories the lowest lying baryons belong to the set of Goldstones of the spontaneously broken extended flavor symmetry $S U\left(2 N_{f}\right)$. As a result the dependence on $\mu$ can be described in the Chiral Perturbation Theory framework [26, 27, 28].

We shall construct the effective Lagrangian governing the low-momentum modes in the theory. These modes are the Goldstone particles of the spontaneously broken global symmetries. The symmetry of the theory is largest at $\mu=0, m=0$, and so is the number of true Goldstone modes. A nonzero chemical potential $\mu$ and/or a bare quark mass $m$ removes 
part of the symmetry and some of the Goldstone modes acquire masses. Our main goal is to find the functional dependence of the masses of such pseudo-Goldstones on $\mu$ and $m$. As we proceed we learn about many other properties of the theory: condensates and vacuum alignment, phase transitions, etc.

The two cases: two-color fundamental quarks $(\beta=1)$ and any-color adjoint quarks $(\beta=4)$ can be analyzed in a similar way. We shall perform this analysis as follows. At each step we shall begin with $\beta=1$ case, explaining the concepts and ideas. Then we follow it immediately with the same analysis for the $\beta=4$ case with emphasis on the comparison between the two cases, which will help understand both cases better. We shall use similar notations for the objects which are conceptually the same in both cases. In many instances we need not rewrite the formulas, only changing the meaning of the notations suffices. All the formulas which use any properties specific to either $\beta=1$ or $\beta=4$ are explicitly tagged. The formulas without such tags are general and apply to both cases. As we shall quickly see the two cases naturally complement each other and are, in a certain sense, dual to each other.

\section{Global symmetries at $m=\mu=0$}

\section{$3.1 \beta=1$}

The fermionic part of the QCD Lagrangian with 2 fundamental colors is given by

$$
\mathcal{L}=\bar{\psi} \gamma_{\nu} D_{\nu} \psi=i\left(\begin{array}{c}
\psi_{L}^{*} \\
\psi_{R}^{*}
\end{array}\right)^{T}\left(\begin{array}{cc}
\sigma_{\nu} D_{\nu} & 0 \\
0 & -\sigma_{\nu}^{\dagger} D_{\nu}
\end{array}\right)\left(\begin{array}{c}
\psi_{L} \\
\psi_{R}
\end{array}\right)
$$

We are working in Euclidean space with hermitian $\gamma$-matrices and we use spin matrices $\sigma_{\nu}=\left(-i, \sigma_{k}\right)$. The quark flavor (as well as color and spin) indices are suppressed and the sum over $N_{f}$ flavors is implied. The symbol $D_{\nu}$ denotes color covariant derivative, $\partial_{\nu}+i A_{\nu}$, an antihermitian operator, with $A_{\nu}$ being a matrix in color algebra, $A_{\nu}=A_{\nu}^{a} \tau_{a} / 2$. As usual $\bar{\psi}=\psi^{\dagger} \gamma_{0}=\psi^{* T} \gamma_{0}$ and in the Euclidean partition function $\psi$ and $\psi^{*}$ are independent integration variables.

The fact that the Lagrangian (3) has a higher flavor symmetry than the apparent $U\left(N_{f}\right) \times$ $U\left(N_{f}\right)$ is related to the pseudoreality of the two-color Dirac operator. In particular, the conjugate field $\tilde{\psi}_{R}=\sigma_{2} \tau_{2} \psi_{R}^{*}$ transforms similarly (in the same color representation) to $\psi_{L}$. Rewriting the Lagrangian (3) we find

$$
\mathcal{L}=i\left(\begin{array}{c}
\psi_{L}^{*} \\
\tilde{\psi}_{R}^{*}
\end{array}\right)^{T}\left(\begin{array}{cc}
\sigma_{\nu} D_{\nu} & 0 \\
0 & \sigma_{\nu} D_{\nu}
\end{array}\right)\left(\begin{array}{c}
\psi_{L} \\
\tilde{\psi}_{R}
\end{array}\right)=i \Psi^{\dagger} \sigma_{\nu} D_{\nu} \Psi
$$

where we have used the well-known properties of the Pauli matrices, $-\sigma_{2} \sigma_{\nu}^{\dagger} \sigma_{2}=\sigma_{\nu}^{T}$ and $-\tau_{2} \tau_{k} \tau_{2}=\tau_{k}^{T}$, taken into account anticommutativity of Grassman variables, dropped total derivatives, and introduced the spinor of dimension $2 N_{f}$,

$$
\Psi \equiv\left(\begin{array}{c}
\psi_{L} \\
\sigma_{2} \tau_{2} \psi_{R}^{*}
\end{array}\right) \equiv\left(\begin{array}{c}
\psi_{L} \\
\widetilde{\psi}_{R}
\end{array}\right) . \quad(\beta=1)
$$

In this form the $U\left(2 N_{f}\right)$ symmetry becomes manifest. Due to the axial anomaly the symmetry in the corresponding quantum theory is only $S U\left(2 N_{f}\right)$ (up to discrete symmetries). 


\section{$3.2 \beta=4$}

QCD with quarks in the adjoint representation of the color group is described by the Lagrangian as in (3), but with different notations. The fields $\psi$ are now transforming according to the adjoint representation of the color group. The color covariant derivative is again given by $D_{\nu}=\partial_{\nu}+i A_{\nu}$, but now $A_{\nu}$ is given by the antisymmetric matrix $\left(A_{\nu}\right)^{b c}=A_{\nu}^{a} f_{a}^{b c}$, where $f_{a}^{b c}=f^{a b c}$ are the generators of the adjoint representation, i.e., the structure constants.

The antisymmetric property of the structure constants now replaces the property of the fundamental generators: $\tau_{2} \tau_{k} \tau_{2}=-\tau_{k}^{T} \rightarrow f_{a}^{b c}=-f_{a}^{c b}$. Using this property we can again recast the Lagrangian using spinors of length $2 N_{f}$ and obtain (州), but with the spinors $\Psi$ (and $\tilde{\psi}_{R}$ ) defined by

$$
\Psi \equiv\left(\begin{array}{c}
\psi_{L} \\
\sigma_{2} \psi_{R}^{*}
\end{array}\right) \equiv\left(\begin{array}{c}
\psi_{L} \\
\tilde{\psi}_{R}
\end{array}\right) . \quad(\beta=4)
$$

The only difference from (5) is the absence of color $\tau_{2}$ matrix in the definition of $\tilde{\psi}_{R}$, and, of course, the fact that the spinors $\psi$ carry an adjoint, instead of a fundamental, color index. Similarly, in terms of the spinors (6) the $S U\left(2 N_{f}\right)$ symmetry of the theory becomes manifest.

\section{Spontaneous symmetry breaking and Goldstones}

\section{$4.1 \beta=1$}

Let us now understand transformation properties of $\langle\bar{\psi} \psi\rangle$, the order parameter of the chiral symmetry breaking, with respect to the $S U\left(2 N_{f}\right)$ symmetry of the theory. We can rewrite $\bar{\psi} \psi$ as

$$
\bar{\psi} \psi=\left(\begin{array}{c}
\psi_{L}^{*} \\
\psi_{R}^{*}
\end{array}\right)^{T}\left(\begin{array}{cc}
0 & 1 \\
1 & 0
\end{array}\right)\left(\begin{array}{c}
\psi_{L} \\
\psi_{R}
\end{array}\right)=\frac{1}{2} \Psi^{T} \sigma_{2} \tau_{2}\left(\begin{array}{cc}
0 & -1 \\
1 & 0
\end{array}\right) \Psi+\text { h.c. } . \quad(\beta=1)
$$

The Pauli matrices $\sigma_{2}$ and $\tau_{2}$ ensure antisymmetrization in spin and color indices, to produce a spin and color singlet. We see that the condensate is not invariant under all $S U\left(2 N_{f}\right)$ rotations. The subgroup which leaves (17) invariant is $S p\left(2 N_{f}\right)$. The Goldstone manifold is therefore given by $S U\left(2 N_{f}\right) / S p\left(2 N_{f}\right)$ with $N_{f}\left(2 N_{f}-1\right)-1$ independent degrees of freedom.

Another way of counting the total number of Goldstone modes is starting from the observation that the condensate ([0) is a product of two fundamental $S U\left(2 N_{f}\right)$ flavor representations, antisymmetric in flavor indices. Therefore, the condensate belongs to an antisymmetric tensor representation the dimension of which is $N_{f}\left(2 N_{f}-1\right)$. Condensation can occur in any of these $N_{f}\left(2 N_{f}-1\right)$ directions; the fluctuations along the remaining $N_{f}\left(2 N_{f}-1\right)-1$ directions then become Goldstone modes.

The effective theory for the Goldstone modes can be written in terms of the fluctuations of the orientation of the chiral condensate, $\Sigma$, which, according to the previous paragraph, is an antisymmetric unimodular (det $\Sigma=1$ ) unitary matrix (exactly $N_{f}\left(2 N_{f}-1\right)-1$ independent components). If we denote the equilibrium value of the orientation of the chiral condensate 
by $\Sigma_{c}$, the Goldstone manifold given by $S U\left(2 N_{f}\right) / S p\left(2 N_{f}\right)$ can be parameterized, according to the transformation of $\Sigma$ under $S U\left(2 N_{f}\right)$, by

$$
\Sigma=U \Sigma_{c} U^{T}
$$

where

$$
U=\exp \left(\frac{i \Pi}{2 F}\right) \text { and } \Pi=\pi_{a} \frac{X_{a}}{\sqrt{2 N_{f}}} .
$$

The fields $\pi_{a}$ are the Goldstone modes. The implied sum (over $a$ ) is over the $N_{f}\left(2 N_{f}-1\right)-1$ generators of the coset $S U\left(2 N_{f}\right) / S p\left(2 N_{f}\right)$ and in order to simplify the algebra in later sections we are using the normalization

$$
\operatorname{Tr} X_{a} X_{b}=2 N_{f} \delta_{a b}
$$

The construction of the Goldstone manifold (9) corresponds to the classification of the $\left(2 N_{f}\right)^{2}-1$ generators of the $S U\left(2 N_{f}\right)$ with respect to a fixed antisymmetric antiunitary matrix, in our case given by $\Sigma_{c}$, into $T_{k}$ and $X_{a}\left[18\right.$. The $T_{k}$ generators leave $\Sigma_{c}$ invariant,

$$
\exp \left(i \phi_{k} T_{k}\right) \Sigma_{c} \exp \left(i \phi_{k} T_{k}\right)^{T}=\Sigma_{c} \text {, i.e. } T_{k} \Sigma_{c}=-\Sigma_{c} T_{k}^{T}
$$

By definition, they are the generators of the symplectic group $S p\left(2 N_{f}\right)$. The remaining generators, $X_{a}$, form the coset $S U\left(2 N_{f}\right) / S p\left(2 N_{f}\right)$. They obey the relation

$$
X_{a} \Sigma_{c}=\Sigma_{c} X_{a}^{T}, \quad \text { and thus } \quad U \Sigma_{c} U^{T}=U^{2} \Sigma_{c} .
$$

The partition of generators in generators of the coset, $X_{a}$, and generators of the invariant subgroup $S p\left(2 N_{F}\right), T_{i}$, depends on the matrix $\Sigma_{c}$. The defining relations (12) are left unaltered by rotation of $\Sigma_{c}$ according to

$$
\Sigma_{c} \rightarrow V \Sigma_{c} V^{T}
$$

where $V$ is an $S U\left(2 N_{f}\right)$ matrix, and a simultaneous rotation of the generators by

$$
X_{a} \rightarrow V X_{a} V^{\dagger}
$$

This means that the set of broken generators, $X_{a}$, changes, if the matrix $\Sigma_{c}$ is changed.

If we use, as we do below, the following choice for $\Sigma_{c}$ :

$$
\Sigma_{c}=\left(\begin{array}{cc}
0 & -1 \\
1 & 0
\end{array}\right), \quad(\beta=1)
$$

the generators $X_{a}$ can be written in the block representation as

$$
\Pi=\left(\begin{array}{ll}
P^{T} & Q \\
Q^{\dagger} & P
\end{array}\right),
$$

with $N_{f} \times N_{f}$ matrices $P$ and $Q$ such that $\operatorname{Tr} P=0, P^{\dagger}=P$, and $Q^{T}=-Q$. It is then easy to see that the number of independent components in $P$ and $Q$ are,

$$
N_{P}=N_{f}^{2}-1 \quad \text { and } \quad N_{Q}=N_{f}\left(N_{f}-1\right) . \quad(\beta=1)
$$




\section{$4.2 \beta=4$}

What are the transformation properties of the chiral condensate with respect to the $S U\left(2 N_{f}\right)$ symmetry in the $\beta=4$ case? We can rewrite $\bar{\psi} \psi$ as

$$
\bar{\psi} \psi=\left(\begin{array}{c}
\psi_{L}^{*} \\
\psi_{R}^{*}
\end{array}\right)^{T}\left(\begin{array}{ll}
0 & 1 \\
1 & 0
\end{array}\right)\left(\begin{array}{l}
\psi_{L} \\
\psi_{R}
\end{array}\right)=\frac{1}{2} \Psi^{T} \sigma_{2}\left(\begin{array}{cc}
0 & -1 \\
-1 & 0
\end{array}\right) \Psi+\text { h.c. } \quad(\beta=4)
$$

The Pauli matrix $\sigma_{2}$ ensures the antisymmetrization with respect to spin indices, to produce a spin singlet. There is no antisymmetrization in color (unlike in the case of fundamental colors $\beta=1$ ) - the color singlet is a symmetric product of two adjoint representations. The Pauli principle now demands that the $S U\left(2 N_{f}\right)$ flavor indices must be symmetric, and they are, as is evident in (18). Therefore the condensate belongs to the symmetric (as opposed to antisymmetric for $\beta=1$ ) second rank tensor representation of $S U\left(2 N_{f}\right)$, which has dimension $N_{f}\left(2 N_{f}+1\right)$. The condensation can occur in any of the $N_{f}\left(2 N_{f}+1\right)$ directions. The fluctuations in the remaining $N_{f}\left(2 N_{f}+1\right)-1$ directions become Goldstone bosons. Alternatively, since the chiral condensate (18) is invariant under $S O\left(2 N_{f}\right)$, the Goldstone manifold is $S U\left(2 N_{f}\right) / S O\left(2 N_{f}\right)$, which gives us the same number of Goldstone modes.

The Goldstone manifold $S U\left(2 N_{f}\right) / S O\left(2 N_{f}\right)$ should now be parameterized by symmetric unimodular unitary matrices $\Sigma$. The Goldstone fields are introduced in the same way as in (8) and (9), i.e., $\Sigma=U \Sigma_{c} U^{T}$, where $\Sigma_{c}$ is now also a symmetric unimodular unitary matrix and the implied sum in (9) is over the generators $X_{a}$ of the coset $S U\left(2 N_{f}\right) / S O\left(2 N_{f}\right)$.

The classification of the generators is also similar to $\beta=1$ case. The generators of the coset, $X_{a}$ obey the commutation relation (12) with a given symmetric unitary matrix, also denoted by $\Sigma_{c}$, whereas the $T_{i}$ generators leave $\Sigma_{c}$ invariant as in (11). The $T_{i}$ are now the generators of an $S O\left(2 N_{f}\right)$ subgroup (as opposed to $S p\left(2 N_{f}\right)$ in the $\beta=1$ case). The remaining generators $X_{a}$ form a coset $S U\left(2 N_{f}\right) / S O\left(2 N_{f}\right)$, which is the Goldstone manifold in this case.

In this case the standard choice for the matrix $\Sigma_{c}$ is

$$
\Sigma_{c}=\left(\begin{array}{cc}
0 & 1 \\
1 & 0
\end{array}\right) . \quad(\beta=4)
$$

With this choice, the matrix $\Pi=\pi_{a} X_{a} / \sqrt{2 N_{f}}$ in the coset can be split into 4 blocks of size $N_{f} \times N_{f}$ as in (16). The matrix $P$ is again hermitian and traceless, but the matrix $Q$ is now symmetric. Therefore, the counting of the independent degrees of freedom in the $\beta=4$ case is

$$
N_{P}=N_{f}^{2}-1 \quad \text { and } \quad N_{Q}=N_{f}\left(N_{f}+1\right) . \quad(\beta=4)
$$

In both cases, $\beta=1$ and $\beta=4$, the kinetic term of the effective Lagrangian describing the Goldstone modes should be invariant under the global $S U\left(2 N_{f}\right)$ group and under Lorentz transformation. The corresponding $S U\left(2 N_{f}\right)$ nonlinear sigma-model is given by

$$
\mathcal{L}_{\text {eff }}=\frac{F^{2}}{2} \operatorname{Tr} \partial_{\nu} \Sigma \partial_{\nu} \Sigma^{\dagger},
$$

where $F$ is the pion decay constant. 


\section{$5 \quad$ Bare quark mass $m$}

\section{$5.1 \beta=1$}

If the bare quark mass $m$ (in this paper, the same for all quarks) is not zero, an explicit $S U\left(2 N_{f}\right)$ breaking term in the effective Lagrangian (21) appears. To determine its form we first rewrite the bare mass term using $S U\left(2 N_{f}\right)$ notations,

$$
m \bar{\psi} \psi=\frac{1}{2} m \Psi^{T} \sigma_{2} \tau_{2}\left(\begin{array}{cc}
0 & -1 \\
1 & 0
\end{array}\right) \Psi+\text { h.c. }=-\frac{1}{2} \Psi^{T} \sigma_{2} \tau_{2} M \Psi+\text { h.c. }, \quad(\beta=1)
$$

where the mass matrix $M$ is given by

$$
M=m \hat{M} \quad \text { and } \quad \hat{M}=\left(\begin{array}{cc}
0 & 1 \\
-1 & 0
\end{array}\right) . \quad(\beta=1)
$$

We see explicitly that the bare mass term is only invariant under an $S p\left(2 N_{f}\right)$ subgroup of $S U\left(2 N_{f}\right)$. The full $S U\left(2 N_{f}\right)$ invariance can be restored if $M$ is also transformed together with $\Psi$ according to

$$
\Psi \rightarrow V \Psi \quad \text { and } \quad M \rightarrow V^{*} M V^{\dagger}
$$

This extended symmetry must be also manifest in the effective theory,

$$
\Sigma \rightarrow V \Sigma V^{T} \quad \text { and } \quad M \rightarrow V^{*} M V^{\dagger}
$$

The lowest order term induced by the quark mass must therefore have the form

$$
\mathcal{L}_{\text {q.mass }}=-G \operatorname{Re} \operatorname{Tr}(M \Sigma)=-m G \operatorname{Re} \operatorname{Tr}(\hat{M} \Sigma) .
$$

One can view $\operatorname{Re} \operatorname{Tr}(\hat{M} \Sigma)$ as a generalized cosine of the angle between unitary matrices $\Sigma$ and $\hat{M}^{\dagger}$. It is maximal when $\Sigma$ is aligned with $\hat{M}^{\dagger}$. Therefore the direction of $\Sigma$ minimizing (26) is given by

$$
\Sigma_{c}=\hat{M}^{\dagger}
$$

which leads us to our choice of $\Sigma_{c}$ (15). The mass term comes with a phenomenological coefficient, which we denote by $G$. It is given by the derivative of the vacuum energy with respect to $m$ and is, therefore, proportional to the chiral condensate in the chiral limit $m \rightarrow 0$ at $\mu=0$ (see Section 12),

$$
G=\frac{\langle\bar{\psi} \psi\rangle_{0}}{2 N_{f}}
$$

The resulting Lagrangian with the mass term,

$$
\mathcal{L}_{\text {eff }}=\frac{F^{2}}{2} \operatorname{Tr} \partial_{\nu} \Sigma \partial_{\nu} \Sigma^{\dagger}-m G \operatorname{Re} \operatorname{Tr}(\hat{M} \Sigma),
$$

is the familiar Chiral Perturbation Theory Lagrangian at lowest order in the momentum expansion [26, 27]. Expanded to second order in the pion fields according to (8), (9), it 
yields a spectrum with $N_{f}\left(2 N_{f}-1\right)-1$ degenerate (pseudo-)Goldstones with masses given by the usual Gell-Mann-Oakes-Renner relation

$$
m_{\pi}^{2}=\frac{m G}{F^{2}} .
$$

We can use this relation to trade $G$ for another parameter, $m_{\pi}$, and write

$$
\mathcal{L}_{\text {eff }}=\frac{F^{2}}{2}\left[\operatorname{Tr} \partial_{\nu} \Sigma \partial_{\nu} \Sigma^{\dagger}-2 m_{\pi}^{2} \operatorname{Re} \operatorname{Tr}(\hat{M} \Sigma) .\right]
$$

The symmetry of the theory is reduced from $S U\left(2 N_{f}\right)$ to $S p\left(2 N_{f}\right)$ by the mass term. Since the chiral condensate does not break any more symmetries in this case we do not have any true Goldstones when $m \neq 0$.

\section{$5.2 \beta=4$}

To determine the form of the term in the effective Lagrangian induced by a small bare quark mass $m$ we rewrite the quark mass term as

$$
m \bar{\psi} \psi=\frac{1}{2} m \Psi^{T} \sigma_{2}\left(\begin{array}{cc}
0 & -1 \\
-1 & 0
\end{array}\right) \Psi+\text { h.c. }=-\frac{1}{2} \Psi^{T} \sigma_{2} M \Psi+\text { h.c. }, \quad(\beta=4)
$$

where we have used the spinors of length $N_{f}$ introduced in section 3. In this case the mass matrix is given by

$$
M=m \hat{M} \quad \text { with } \quad \hat{M}=\left(\begin{array}{ll}
0 & 1 \\
1 & 0
\end{array}\right) . \quad(\beta=4)
$$

Using the same arguments as in section 5.1 we find that the mass term in the effective Lagrangian as dictated by the extended flavor symmetry is given again by (26), with $\Sigma$ now being a symmetric matrix and $M$ given by (33). Similarly, this term is minimized when $\Sigma=\hat{M}^{\dagger}$. With $\hat{M}$ now taken from (33) we arrive at our choice of $\Sigma_{c}(19)$.

The symmetry of the theory is reduced from $S U\left(2 N_{f}\right)$ to $S O\left(2 N_{f}\right)$ by the mass term. Since the chiral condensate does not break any more symmetries in this case we do not have any truly massless Goldstones when $m \neq 0$. The masses of all $N_{f}\left(2 N_{f}+1\right)$ (pseudo-)Goldstones are equal and are given by the Gell-Mann-Oakes-Renner relation (30).

\section{Chemical potential $\mu$}

In this section we review the introduction of the chemical potential in the effective partition function following the approach of [8]. This approach relies only on the $S U\left(2 N_{f}\right)$ symmetry of the theory and the resulting $\mu$-dependent terms are the same for both cases, $\beta=1$ and $\beta=4$. 


\subsection{Global symmetries and $\mu$}

At nonzero chemical potential the microscopic Lagrangian is given by

$$
\mathcal{L}=\bar{\psi} \gamma_{\nu} D_{\nu} \psi-\mu \bar{\psi} \gamma_{0} \psi+m \bar{\psi} \psi
$$

As was the case for the mass term, we can also rewrite the baryon charge density in terms of the $S U\left(2 N_{f}\right)$ spinors.

$$
\begin{aligned}
& \bar{\psi} \gamma_{0} \psi=\left(\begin{array}{c}
\psi_{L}^{*} \\
\psi_{R}^{*}
\end{array}\right)^{T}\left(\begin{array}{ll}
1 & 0 \\
0 & 1
\end{array}\right)\left(\begin{array}{l}
\psi_{L} \\
\psi_{R}
\end{array}\right)=\Psi^{\dagger}\left(\begin{array}{cc}
1 & 0 \\
0 & -1
\end{array}\right) \Psi \equiv \Psi^{\dagger} B \Psi \\
& B \equiv\left(\begin{array}{cc}
+1 & 0 \\
0 & -1
\end{array}\right) .
\end{aligned}
$$

The physical meaning of +1 and -1 in the baryon charge matrix $B$ is simple: they are the baryon charges of the quarks $\psi_{L}$ and conjugate quarks $\psi_{R}$. We see that this term is not a singlet under $S U\left(2 N_{f}\right)$. It transforms in the adjoint representation of this group, in other words, the baryon charge is one of the $\left(2 N_{f}\right)^{2}-1$ generators of this group. In terms of the spinors of length $2 N_{f}$, the microscopic Lagrangian is thus given by

$$
\mathcal{L}=i \Psi^{\dagger} \sigma_{\nu}\left(D_{\nu}-\mu B_{\nu}\right) \Psi-\left[\frac{1}{2} \Psi^{T}\left\{\begin{array}{c}
\sigma_{2} \tau_{2} \\
\sigma_{2}
\end{array}\right\} M \Psi+\text { h.c. }\right],
$$

where the upper branch corresponds to $\beta=1$ and the lower branch to $\beta=4$. For reasons that will become clear in the next subsection, we have introduced the four-vector $B_{\nu}=(B, \mathbf{0})$.

As in the case of the quark mass term, the chemical potential term, $\mu \Psi^{\dagger} B \Psi$ violates the $S U\left(2 N_{f}\right)$ symmetry. Similarly, we can maintain this symmetry by accompanying the rotation of $\Psi$ by a corresponding rotation of $B$,

$$
\Psi \rightarrow V \Psi \text { and } B \rightarrow V B V^{\dagger} .
$$

Such an extended symmetry must be manifest in the effective Lagrangian,

$$
\Sigma \rightarrow V \Sigma V^{T} \text { and } B \rightarrow V B V^{\dagger} .
$$

This restricts the lowest order nonderivative term in $\mu$ to a linear combination of

$$
\mu^{2} \operatorname{Tr}\left(\Sigma B^{T} \Sigma^{\dagger} B\right) \quad \text { and } \quad \mu^{2} \operatorname{Tr}(B B)
$$

with arbitrary coefficients. Only the first of these terms contains a dependence on the Goldstone fields.".

At $m=0$ the chemical potential breaks the global symmetry of the theory from $S U\left(2 N_{f}\right)$ down to the usual (as in three-color QCD) $S U\left(N_{f}\right)_{L} \times S U\left(N_{f}\right)_{R} \times U(1)_{B}$. If $m \neq 0$ also, the symmetry of the theory is only $S U\left(N_{f}\right)_{V} \times U(1)_{B}$.

\footnotetext{
${ }^{1}$ Such type of symmetry breaking terms also occur in the context of the non-hermitian Random Matrix Theory [7, 29, 30].
} 


\subsection{Local symmetry and the coefficient of the $\mu^{2}$ term}

The coefficients of the terms (39) in the effective Lagrangian can be related to baryon number susceptibility, i.e., the second derivative of the vacuum energy with respect to $\mu$. However, unlike $G$, i.e. the chiral condensate $\langle\bar{\psi} \psi\rangle_{0}$, these parameters are not independent. They are related to the pion decay constant $F$ by virtue of a local symmetry [8].

As was observed in [8], one can further extend the symmetry (37) of the microscopic Lagrangian (36) to include local $S U\left(2 N_{f}\right)$ flavor transformations

$$
\Psi \rightarrow V \Psi \quad \text { and } \quad B_{\nu} \rightarrow V B_{\nu} V^{\dagger}-\frac{1}{\mu} V \partial_{\nu} V^{\dagger}
$$

We can also recover the Lorentz (Euclidean) symmetry by transforming $B_{\nu}$ as a four-vector.

To make such an extended local symmetry (and also Lorentz symmetry) manifest in the effective Lagrangian (31) we must replace the normal derivative by a flavor covariant derivative,

$$
\begin{aligned}
& \nabla_{\nu} \Sigma=\partial_{\nu} \Sigma-\mu\left(B_{\nu} \Sigma+\Sigma B_{\nu}^{T}\right) \\
& \nabla_{\nu} \Sigma^{\dagger}=\partial_{\nu} \Sigma^{\dagger}+\mu\left(\Sigma^{\dagger} B_{\nu}+B_{\nu}^{T} \Sigma^{\dagger}\right) .
\end{aligned}
$$

Thus we arrive at the effective Lagrangian of lowest order in the momentum expansion

$$
\begin{aligned}
\mathcal{L}_{\text {eff }}= & \frac{F^{2}}{2}\left[\operatorname{Tr} \nabla_{\nu} \Sigma \nabla_{\nu} \Sigma^{\dagger}-2 m_{\pi}^{2} \operatorname{Re} \operatorname{Tr}(\hat{M} \Sigma)\right] \\
= & \frac{F^{2}}{2} \operatorname{Tr} \partial_{\nu} \Sigma \partial_{\nu} \Sigma^{\dagger}+2 \mu F^{2} \operatorname{Tr} B \Sigma^{\dagger} \partial_{0} \Sigma \\
& -F^{2} \mu^{2} \operatorname{Tr}\left(\Sigma B^{T} \Sigma^{\dagger} B+B B\right)-F^{2} m_{\pi}^{2} \operatorname{Re} \operatorname{Tr}(\hat{M} \Sigma) .
\end{aligned}
$$

It is important to note that the dependence on $\mu$ comes with no additional parameters. It is completely fixed, by the local symmetry, in terms of an already existing parameter $F$.

\section{Vacuum alignment}

The static part of the Lagrangian (42) determines the vacuum alignment of the field $\Sigma$ as well as the masses of the excitations. This part of the Lagrangian has the form

$$
\begin{aligned}
\mathcal{L}_{\mathrm{st}}(\Sigma) & =-F^{2} \mu^{2} \operatorname{Tr}\left(\Sigma B^{T} \Sigma^{\dagger} B+B B\right)-F^{2} m_{\pi}^{2} \operatorname{Re} \operatorname{Tr}(\hat{M} \Sigma) \\
& =\frac{F^{2} m_{\pi}^{2}}{2}\left[-\frac{x^{2}}{2} \operatorname{Tr}\left(\Sigma B^{T} \Sigma^{\dagger} B+B B\right)-2 \operatorname{Re} \operatorname{Tr}(\hat{M} \Sigma)\right],
\end{aligned}
$$

where we introduced $x=2 \mu / m_{\pi}$. The $\mu^{2}$ and $m_{\pi}^{2}$ terms in (43) compete for the direction of the condensation which we denote by $\bar{\Sigma}$. For $x=0$ the orientation of $\bar{\Sigma}$ is determined by the mass matrix $\bar{\Sigma}=\hat{M}^{\dagger}$. This value we denoted by $\Sigma_{c}$. This is the orientation of the usual chiral condensate which carries no baryon charge. When $x=\infty$ [8] the static Lagrangian is 
minimized on a manifold in the space of $\Sigma$, from which we choose the following value and denote it by $\Sigma_{d}$,

$$
\Sigma_{d}=\left(\begin{array}{cc}
i I & 0 \\
0 & i I
\end{array}\right), \quad \quad(\beta=1)
$$

where $I$ is an antisymmetric $N_{f} \times N_{f}$ matrix, which, written in $\left(N_{f} / 2\right) \times\left(N_{f} / 2\right)$ blocks, has the form

$$
I=\left(\begin{array}{cc}
0 & -1 \\
1 & 0
\end{array}\right)
$$

This minimum (even for $m \neq 0$, unlike $\Sigma_{c}$ ) has a degeneracy, which for $N_{f}=2$, is simply a rotation by the generator $B$. The condensate $\Sigma_{d}$ breaks spontaneously the baryon number symmetry. The degeneracy leads to the appearance of a massless Goldstone boson. For $N_{f}>2$ this condensate breaks more than just the $U(1)_{B}$ symmetry. For $m=0$ it also breaks $S U\left(N_{f}\right)_{L} \times S U\left(N_{f}\right)_{R}$ down to $S p\left(N_{f}\right)_{L} \times S p\left(N_{f}\right)_{R}$ given by the rotations leaving the block matrix $I$ in (44) invariant [8]. When $m \neq 0$ it breaks the $S U\left(N_{f}\right)_{V}$ symmetry down to $\operatorname{Sp}\left(N_{f}\right)_{V} \cdot$ ? $^{2}$

The situation is similar in the $\beta=4$ case. The only difference is that now the matrix $\Sigma$ is a symmetric $S U\left(2 N_{f}\right)$ matrix. It rotates between the value of $\Sigma_{c}$ given by (19) and $\Sigma_{d}$, which we chose as

$$
\Sigma_{d}=\left(\begin{array}{cc}
i & 0 \\
0 & i
\end{array}\right) . \quad(\beta=4)
$$

The discussion of the preceding paragraph carries over to the $\beta=4$ case with the already familiar substitution of $S p\left(N_{f}\right)$ by $S O\left(N_{f}\right)$. The condensate $\Sigma_{d}$ breaks $S U\left(N_{f}\right)_{L, R}$ flavor symmetries down to $S O\left(N_{f}\right)_{L, R}$, in addition to breaking $U(1)_{B}$.

At intermediate values of $x$ the orientation of the condensate $\bar{\Sigma}$ rotates, as a function of $x$, from $\Sigma_{c}$ to $\Sigma_{d}$. We shall prove that it can always be written as the linear combination

$$
\bar{\Sigma}=\Sigma_{\alpha} \equiv \Sigma_{c} \cos \alpha+\Sigma_{d} \sin \alpha,
$$

where $\alpha=0$ for $x=0$ and $\pi / 2$ for $x=\infty$.

The angle $\alpha$ is a function of $x$. It is determined by substituting the value of $\Sigma$ given by (47) into (43). This results in the static Lagrangian

$$
\mathcal{L}_{\mathrm{st}}\left(\Sigma_{\alpha}\right)=F^{2} m_{\pi}^{2} N_{f}\left[\frac{x^{2}}{2}(\cos 2 \alpha-1)-2 \cos \alpha\right] .
$$

Minimizing with respect to $\alpha$ we find

$$
\begin{aligned}
\alpha=0, & \text { when } x<1 ; \\
\cos \alpha=\frac{1}{x^{2}}, & \text { when } x>1 .
\end{aligned}
$$

\footnotetext{
${ }^{2}$ Similarly to the alignment of $\Sigma_{c}$ to the bare quark mass matrix $M^{\dagger}$, the direction of $\Sigma_{d}$ is determined by an external diquark source, $J$, which, as we shall see in section 11, breaks the degeneracy. When $J$ is zero, we can choose any orientation of $\Sigma_{d}$ within the manifold of minima, and the results will not change due to the symmetry relating all such minima.
} 
Note that $\alpha=0$ is always an extremum of (48) but it becomes a maximum for $x>1$.

We find that the condensate is a non-analytic function of $x=2 \mu / m_{\pi}$. There is a second order phase transition as a function of $\mu$ at $x=1$. For small $\mu<m_{\pi} / 2$ the vacuum does not change. At $\mu=m_{\pi} / 2$ a transition occurs and the direction of the condensate starts rotating from that of $\Sigma_{c}$ to that of $\Sigma_{d}$. A nonzero value of the projection on $\Sigma_{d}$, proportional to $\sin \alpha$, means that the vacuum breaks the baryon number symmetry spontaneously. This happens at the value of $\mu$ equal to $1 / 2$ of the mass of the lightest baryon in the system - the diquark, or, the baryonic pion.

\section{Global minimum}

In this section we show that the minimum of the static potential is given by $\Sigma_{\alpha}$ defined in (47). The argument in this section shows that it is an absolute, or global, minimum of $\mathcal{L}_{\text {st }}(\Sigma)$. In the next section we expand the Goldstone fields to second order about this minimum.

\section{$8.1 \beta=1$}

We decompose the antisymmetric unitary matrix $\Sigma$ into 4 blocks of size $N_{f} \times N_{f}$,

$$
\Sigma=\left(\begin{array}{cc}
A & -C \\
C^{T} & B
\end{array}\right) . \quad(\beta=1)
$$

The antisymmetric matrices $A$ and $B$ satisfy the following unitarity constraints:

$$
A A^{\dagger}+C C^{\dagger}=1, \quad B B^{\dagger}+\left(C^{\dagger} C\right)^{T}=1, \quad A C^{*}=C B^{\dagger} . \quad(\beta=1)
$$

Taking this into account we can express $\mathcal{L}_{\text {st }}$ entirely in terms of the matrix $C$,

$$
\mathcal{L}_{\text {st }}(\Sigma)=F^{2} m_{\pi}^{2}\left[x^{2} \operatorname{Tr}\left(C-\frac{1}{x^{2}}\right)\left(C^{\dagger}-\frac{1}{x^{2}}\right)-N_{f}\left(x^{2}+\frac{1}{x^{2}}\right)\right] .
$$

Ignore, for the moment, the constraints on the matrix elements of $C$. The trace in (52) can be viewed as the distance in the $2 N_{f}^{2}$ dimensional space of real and imaginary parts of the matrix elements of $C$ from the point given by diagonal matrix $1 / x^{2}$. For $x>1$ the absolute minimum is achieved when $C=1 / x^{2}$. The matrices $A$ and $B$ can be chosen, for example,

as $A=B=i I \sqrt{1-1 / x^{4}}$, to satisfy all the constraints (51). If we define $\cos \alpha=1 / x^{2}$, we observe that the resulting matrix in (50) is given by $\Sigma_{\alpha}(47)$.

When $x<1$, we notice that unitarity constraints (51) demand that $\operatorname{Tr} C C^{\dagger} \leq 1$. This means that we have to consider the points in the space of $C$ only within the unit hypersphere around $C=0$. It is easy to see that the minimum distance within this sphere is at the surface point closest to $1 / x^{2}$, i.e. at $C=1$. The constraints (51) can be satisfied only by $A=B=0$ and the resulting matrix in (50) is $\Sigma_{c}$, the minimum of the static potential at $\mu=0$. 


\section{$8.2 \beta=4$}

The same analysis applies in the $\beta=4$ case. Since $\Sigma$ is now a symmetric matrix its block decomposition is given by

$$
\Sigma=\left(\begin{array}{cc}
A & +C \\
C^{T} & B
\end{array}\right), \quad(\beta=4)
$$

with symmetric matrices $A$ and $B$ and with unitarity constraints

$$
A A^{\dagger}+C C^{\dagger}=1, \quad B B^{\dagger}+\left(C^{\dagger} C\right)^{T}=1, \quad A C^{*}=-C B^{\dagger} . \quad(\beta=4)
$$

The static part of the Lagrangian is the same as in (52). The minimum is given by $C=1$ for $x<1$ and $C=1 / x^{2}$ for $x>1$. The matrices $A$ and $B$ are not determined uniquely by

the unitarity constraints. One can check that $A=B=i \sqrt{1-1 / x^{4}}$ (as in (47)) satisfy these constraints. [3

\section{Curvatures at the minimum}

We have established that the global minimum of the effective Lagrangian is achieved at the value of the field $\Sigma$ given by (47) and (49). The orientation of the condensate rotates as a function of $x=2 \mu / m_{\pi}$ (nonanalytic at $x=1$ ). In this section we shall expand the effective Lagrangian up to the second order in the fluctuations of $\Sigma$ which will help us to determine the (pseudo-) Goldstone masses and their dependence on $x$.

\subsection{Normal phase: $\mu<m_{\pi} / 2$}

When $x<1$ the vacuum orientation of $\Sigma$ does not depend on $x$ and is given by $\bar{\Sigma}=\Sigma_{c}$. Expanding $\Sigma$ around $\Sigma_{c}$ using the Goldstone fields defined in (9) according to

$$
\Sigma=U \Sigma_{c} U^{T}=U^{2} \Sigma_{c}=\left(1+\frac{i \Pi}{F}-\frac{\Pi^{2}}{2 F^{2}}+\ldots\right) \Sigma_{c}
$$

\footnotetext{
3 As in the case of $\beta=1$, the case of odd number of flavors has to be investigated separately. Let us consider $N_{f}=1$. Then a symmetric unitary matrix has two degrees of freedom and can be parameterized as

$$
\Sigma=\left(\begin{array}{cc}
i \sin \theta e^{i \phi} & \cos \theta \\
\cos \theta & i \sin \theta e^{-i \phi}
\end{array}\right)
$$

Notice that the determinant of $\Sigma$ has to be equal to the determinant of the mass matrix $\hat{M}(33)$. We thus have that the matrix $C$ in (53) is just a number parameterized according to $C=\cos \theta$, leading to the effective potential (up to constants)

$$
x^{2} \operatorname{Tr}\left(C^{\dagger}-\frac{1}{x^{2}}\right)\left(C-\frac{1}{x^{2}}\right)-x^{2}-\frac{1}{x^{2}}=x^{2} \cos ^{2} \theta-2 \cos \theta-x^{2} .
$$

The minimum is at $\theta=0$ for $x<1$ and at $\cos \theta=1 / x^{2}$ for $x>1$. In the diquark condensation phase $(x>1)$, we find one massless excitation (the $\phi$-mode), a nonzero baryon density and nonzero chiral and diquark condensates.
} 
we find

$$
\mathcal{L}_{\text {st }}(\Sigma)=\mathcal{L}_{\text {st }}\left(\Sigma_{c}\right)+\frac{m_{\pi}^{2}}{2}\left[\frac{x^{2}}{4} \operatorname{Tr}[B, \Pi]^{2}+\operatorname{Tr} \Pi^{2}\right]+\ldots,
$$

where ellipsis denotes terms of higher powers of $\Pi$. The commutator in this Lagrangian can be written as

$$
[B, \Pi]=b \Pi,
$$

with $b$ the baryon charge of the pseudo-Goldstone modes. Since all our pseudo-Goldstone modes are quark-antiquark or (anti-)diquark states the values of $b$ are $b=0, \pm 2$. The curvature of the $P, Q$ and $Q^{\dagger}(16)$ modes, thus depends on $\mu$ through the baryon charge and is given by $m_{\pi}^{2}-(b \mu)^{2}$. For example, in the $\beta=1$ case of $N_{f}=2$, there are 3 pseudoscalar mesons, 1 diquark and 1 antidiquark. Using the block decomposition of the generators (16) we find

$$
\mathcal{L}_{\mathrm{st}}(\Sigma)=\mathcal{L}_{\mathrm{st}}\left(\Sigma_{c}\right)+m_{\pi}^{2} \operatorname{Tr}\left[P^{2}+\left(1-x^{2}\right) Q Q^{\dagger}\right]+\ldots
$$

Two comments are in order here. First, note that, the curvature (and the mass) of the meson modes $P$ does not depend on $\mu$ in the normal phase. Second, at $x=1$ the curvature of the diquark modes $Q$ vanish, which signals a phase transition and the onset of diquark condensation.

\subsection{Diquark condensation phase: $\mu>m_{\pi} / 2$.}

In this phase the condensate begins to rotate according to (47), (49). This rotation can be also written as

$$
\Sigma_{\alpha}=V_{\alpha} \Sigma_{c} V_{\alpha}^{T}=V_{\alpha}^{2} \Sigma_{c}, \text { where } V_{\alpha}^{2}=e^{i \alpha X_{2}},
$$

and $X_{2}$ is the generator that rotates $\Sigma_{c}$ into $\Sigma_{d}$. Comparing (59) (47) we find

$$
\Sigma_{d}=i X_{2} \Sigma_{c}
$$

This generator belongs to the set of broken generators with respect to $\Sigma_{c}$ (as well as with respect to $\Sigma_{d}$ ) since it satisfies (12).

We could parameterize fluctuations of $\Sigma$ around the vacuum value given by $\Sigma_{\alpha}$ as

$$
\Sigma=U_{\alpha} \Sigma_{\alpha} U_{\alpha}^{T}
$$

where $U_{\alpha}$ are unitary matrices generated by rotated (in the sense of (14)) generators, $V_{\alpha} X_{a} V_{\alpha}^{\dagger}$, instead of $X_{a}$ (8), (9). However, as we shall find, the meson mass matrix is diagonal in the basis given by the parametrization

$$
\Sigma=V_{\alpha} U \Sigma_{c} U^{T} V_{\alpha}^{T},
$$

where we have rotated the coset generators $X_{a}$ back to their $\alpha=0$ values using (13), (14) and (59). 
Before expanding the static Lagrangian, we substitute (62) into (43) to obtain

$$
\begin{aligned}
\mathcal{L}_{\mathrm{st}}(\Sigma)=\frac{F^{2} m_{\pi}^{2}}{2}[ & -\frac{x^{2}}{2} \operatorname{Tr}\left(U^{2} \Sigma_{c}\left(V_{\alpha}^{\dagger} B V_{\alpha}\right)^{T} \Sigma_{c}^{\dagger}\left(U^{\dagger}\right)^{2}\left(V_{\alpha}^{\dagger} B V_{\alpha}\right)+B B\right) \\
& \left.-2 \operatorname{Re} \operatorname{Tr}\left(\left(V_{\alpha}^{T} \hat{M} V_{\alpha}\right) U^{2} \Sigma_{c}\right)\right] .
\end{aligned}
$$

The rotated values of $B$ and $\Sigma_{c}$ (by angle $-\alpha$ in the sense of (59) ) can be expressed as

$$
\begin{aligned}
& V_{\alpha}^{\dagger} B V_{\alpha}=V_{-\alpha} B V_{-\alpha}^{\dagger}=B \cos \alpha+X_{1} \sin \alpha ; \\
& V_{\alpha}^{T} \hat{M} V_{\alpha}=\left(V_{-\alpha} \hat{M}^{\dagger} V_{-\alpha}^{T}\right)^{\dagger}=\Sigma_{-\alpha}^{\dagger}=\Sigma_{c}^{\dagger} \cos \alpha-\Sigma_{d}^{\dagger} \sin \alpha
\end{aligned}
$$

where we have introduced $X_{1}$ by

$$
X_{1}=i B X_{2} .
$$

This generator, similarly to $X_{2}$, belongs to the coset of broken generators with respect to $\Sigma_{c}$ (12). It is the generator into which $B$ is rotated while $\Sigma_{c}$ is rotated into $\Sigma_{d}$.

Substituting (64) into (63) and expanding to second order in $\Pi$ (55) we find

$$
\begin{aligned}
\mathcal{L}_{\mathrm{st}}(\Sigma)= & \mathcal{L}_{\mathrm{st}}\left(\Sigma_{\alpha}\right)+F m_{\pi}^{2}\left[-\frac{x^{2}}{2} \sin 2 \alpha+\sin \alpha\right] \operatorname{Tr}\left(X_{2} \Pi\right) \\
& +\frac{m_{\pi}^{2}}{2}\left[\frac{x^{2}}{4}\left(\operatorname{Tr}[B, \Pi]^{2} \cos ^{2} \alpha-\operatorname{Tr}\left[X_{1}, \Pi\right]^{2} \sin ^{2} \alpha\right)+\operatorname{Tr} \Pi^{2} \cos \alpha\right]+\ldots
\end{aligned}
$$

As should be expected, the linear term vanishes due to (49) and we shall concentrate now on the quadratic term.

\subsection{1 $\beta=1$}

At this point we need the explicit form of $X_{1}$ for $\beta=1$. According to (60) we have

$$
X_{2}=-i \Sigma_{d} \Sigma_{c}^{\dagger}=\left(\begin{array}{cc}
0 & I \\
-I & 0
\end{array}\right) . \quad(\beta=1)
$$

For $X_{1}$ we therefore find

$$
X_{1}=i B X_{2}=\left(\begin{array}{cc}
0 & i I \\
i I & 0
\end{array}\right) . \quad(\beta=1)
$$

We then use the block decomposition of $\Pi(16)$ and find

$$
\begin{aligned}
\mathcal{L}_{\mathrm{st}}(\Sigma)=\mathcal{L}_{\mathrm{st}}\left(\Sigma_{\alpha}\right)+m_{\pi}^{2} \operatorname{Tr}\left[-x^{2}\left(Q Q^{\dagger} \cos ^{2} \alpha-\left(P_{S}^{2}+Q_{R} Q_{R}^{\dagger}\right) \sin ^{2} \alpha\right)\right. & \\
& \left.+\left(P^{2}+Q Q^{\dagger}\right) \cos \alpha\right]+\ldots \quad(\beta=1)
\end{aligned}
$$

We have introduced the following projections of $P$ and $Q$ :

$$
\begin{aligned}
& P_{S}=\frac{1}{2}\left(P+I P^{T} I\right) \quad \text { and } \quad P_{A}=\frac{1}{2}\left(P-I P^{T} I\right) ; \\
& Q_{R}=\frac{1}{2}\left(Q+I Q^{\dagger} I\right) \quad \text { and } \quad Q_{I}=\frac{1}{2}\left(Q-I Q^{\dagger} I\right) . \quad(\beta=1)
\end{aligned}
$$


These projections are orthogonal, $\operatorname{Tr} P_{S} P_{A}=\operatorname{Re} \operatorname{Tr} Q_{R} Q_{I}^{\dagger}=0$. Using this fact and the relation $\cos \alpha=1 / x^{2}$ we obtain

$$
\mathcal{L}_{\mathrm{st}}(\Sigma)=\mathcal{L}_{\mathrm{st}}\left(\Sigma_{\alpha}\right)+m_{\pi}^{2} x^{2} \operatorname{Tr}\left[Q_{R} Q_{R}^{\dagger} \sin ^{2} \alpha+P_{A}^{2} \cos ^{2} \alpha+P_{S}^{2}\right]+\ldots \quad(\beta=1)
$$

From (71) we can now read off the curvatures for the different multiplets of the (pseudoGoldstone modes. We see that there is a true flat direction, $Q_{I}$, which describes true massless Goldstones of the diquark condensation phase. These fields are the phases (a single $U(1)_{B}$ phase for $N_{f}=2$ and a set of $U(1)_{B} \times S U\left(N_{f}\right)_{V} / S p\left(N_{f}\right)_{V}$ phases for other $\left.N_{f}\right)$ of the diquark condensate. As we shall see in the next section, the linear derivative terms in the effective Lagrangian mix $Q_{I}$ and $Q_{R}$, thus the actual true Goldstone excitations are certain linear combinations of $Q_{I}$ and $Q_{R}$.

The degeneracies of the multiplets follow from the definitions (70) and are given by

$$
\begin{array}{rlrl}
N_{P_{S}} & =\frac{N_{f}\left(N_{f}+1\right)}{2} ; \quad N_{P_{A}}=\frac{N_{f}\left(N_{f}-1\right)}{2}-1 ; & \\
N_{Q_{R}}=N_{Q_{I}}=\frac{N_{f}\left(N_{f}-1\right)}{2} . & (\beta=1)
\end{array}
$$

They correspond to representations of the group $S p\left(N_{f}\right)$, which is the residual symmetry remaining intact after spontaneous breaking. The number of the flat directions $Q_{I}$ exactly matches the number of the broken generators in $S U\left(N_{f}\right)_{V} \times U(1)_{B} \rightarrow S p\left(N_{f}\right)_{V}$.

\subsection{2 $\beta=4$}

The derivation of the curvatures of the effective potential $\mathcal{L}_{\text {st }}$ in the $\beta=4$ case follows the same lines as in the $\beta=1$ case. All the differences stem from the fact that $\Sigma$ is a symmetric unitary matrix in this case. Consequently, $\Sigma_{c}$ and $\Sigma_{d}$ are given by (19) and (46) instead of (15) and (44). In particular, the generator which rotates $\Sigma_{c}$ into $\Sigma_{d}$ is now given by

$$
X_{2}=-i \Sigma_{d} \Sigma_{c}^{\dagger}=\left(\begin{array}{ll}
0 & 1 \\
1 & 0
\end{array}\right), \quad(\beta=4)
$$

and the generator $X_{1}$, into which $B$ rotates and which is responsible for the splitting of both $P$ and $Q$ branches (in the block decomposition (16)), is given by

$$
X_{1}=i B X_{2}=\left(\begin{array}{cc}
0 & i \\
-i & 0
\end{array}\right) . \quad(\beta=4)
$$

It is now straightforward to rewrite the quadratic part of the Lagrangian (66) in terms of the $P Q$-block decomposition. The mass matrix becomes diagonal in terms of the following projections of $P$ and $Q$ :

$$
\begin{aligned}
P_{S} & =\frac{1}{2}\left(P+P^{T}\right) \quad \text { and } & P_{A} & =\frac{1}{2}\left(P-P^{T}\right) ; \\
Q_{R} & =\frac{1}{2}\left(Q+Q^{\dagger}\right) \quad \text { and } & Q_{I} & =\frac{1}{2}\left(Q-Q^{\dagger}\right) . \quad(\beta=4)
\end{aligned}
$$


The static part of the effective Lagrangian in terms of these fields is given by

$$
\mathcal{L}_{\mathrm{st}}(\Sigma)=\mathcal{L}_{\mathrm{st}}\left(\Sigma_{\alpha}\right)+m_{\pi}^{2} x^{2} \operatorname{Tr}\left[Q_{R} Q_{R}^{\dagger} \sin ^{2} \alpha+P_{S}^{2} \cos ^{2} \alpha+P_{A}^{2}\right]+\ldots, \quad(\beta=4)
$$

which is similar to (71), but $S$ and $A$ labels exchange places.

The main difference is in the dimension of the degenerate multiplets which matches the representations of the corresponding residual symmetry group. By inspection one finds

$$
\begin{array}{rlr}
N_{P_{A}}=\frac{N_{f}\left(N_{f}-1\right)}{2} ; \quad N_{P_{S}}=\frac{N_{f}\left(N_{f}+1\right)}{2}-1 ; & \\
N_{Q_{R}}=N_{Q_{I}}=\frac{N_{f}\left(N_{f}+1\right)}{2} . & (\beta=4)
\end{array}
$$

\section{Spectrum}

In order to complete our program and determine the spectrum of low-lying excitations we must take into account the derivative terms in the Lagrangian (42). They contribute in a non-trivial way to the quadratic form in the Goldstone fields. Due to the non-Lorentz invariant nature of the system we study at finite $\mu$, the dispersion laws do not have a simple form, $E^{2}=\boldsymbol{p}^{2}+m^{2}$. The mass, as measured on the lattice and given by the exponential fall-off of the propagator at large Euclidean time, is the value of $E$, i.e. $i p_{0}$, at the pole of the propagator at $\boldsymbol{p}=0$. These pole masses, or rest energies, we shall now evaluate.

\subsection{Normal phase: $\mu<m_{\pi} / 2$}

Expanding the derivative terms in the Lagrangian (42) in the same way as we expanded the static part in the previous section we obtain

$$
\mathcal{L}(\Sigma)=\mathcal{L}\left(\Sigma_{c}\right)+\frac{1}{2} \operatorname{Tr}\left\{\left(\partial_{\nu} \Pi-\mu\left[B_{\nu}, \Pi\right]\right)^{2}+m_{\pi}^{2} \Pi^{2}\right\}+\ldots
$$

where $B_{\nu}=B \delta_{\nu 0}$, as defined earlier in Section 6.2. Using the Fourier decomposition of $\Pi$,

$$
\Pi(x)=\sum_{p} \Pi_{p} \exp (-i p x)=\sum_{p} \Pi_{p} \exp \left(-E x_{0}-i \boldsymbol{p x}\right),
$$

we find that the dispersion law has the generic form

$$
(E+b \mu)^{2}=\boldsymbol{p}^{2}+m_{\pi}^{2}, \quad \text { or } \quad E=-b \mu+\sqrt{\boldsymbol{p}^{2}+m_{\pi}^{2}}
$$

where $b$ is the baryon charge of the given excitation. This is in agreement with the fact that the effect of $\mu$ on each state is simply an energy shift by $-b \mu$. In particular, the dispersion law of the $P$-type Goldstone modes, which carry no baryon charge, are not affected by $\mu$ at

\footnotetext{
${ }^{4}$ In Euclidean field theory the dispersion relation is given by the poles of the propagator in the $E \equiv i p_{0^{-}}$ plane.
} 
all. The rest energies of the diquarks $Q$ and antidiquarks $Q^{\dagger}$ are shifted according to their charges. To summarize, the dispersion laws in the normal phase are given by

$$
\begin{aligned}
P & : E=\left(m_{\pi}^{2}+\boldsymbol{p}^{2}\right)^{1 / 2} ; \\
Q^{\dagger} & : E=\left(m_{\pi}^{2}+\boldsymbol{p}^{2}\right)^{1 / 2}+2 \mu ; \\
Q & : E=\left(m_{\pi}^{2}+\boldsymbol{p}^{2}\right)^{1 / 2}-2 \mu .
\end{aligned}
$$

The masses of these excitations as well as their degeneracies are given in Tables 1 and 2 . A schematic picture of the mass dependence is shown in Figures 11 and 2.

\subsection{Diquark condensation phase: $\mu>m_{\pi} / 2$.}

In this phase the ground state changes and we need to expand around the rotated value of the condensate $\Sigma=\Sigma_{\alpha}$. We find

$$
\begin{aligned}
\mathcal{L}(\Sigma)=\mathcal{L}\left(\Sigma_{\alpha}\right) & +\frac{1}{2} \operatorname{Tr}\left\{\left(\partial_{\nu} \Pi-\mu\left[V_{\alpha} B_{\nu} V_{\alpha}^{\dagger}, \Pi\right]\right)\left(\partial_{\nu} \Pi-\mu\left[V_{\alpha}^{\dagger} B_{\nu} V_{\alpha}, \Pi\right]\right)\right. \\
& \left.+m_{\pi}^{2} \Pi^{2} \cos \alpha\right\}+\ldots
\end{aligned}
$$

Expanding the product we find

$$
\mathcal{L}(\Sigma)=\frac{1}{2} \operatorname{Tr}\left\{\left(\partial_{\nu} \Pi\right)^{2}-2 \mu \cos \alpha[B, \Pi] \partial_{0} \Pi\right\}+\mathcal{L}_{\text {stat }}(\Sigma)+\ldots
$$

with the static part given by (66). We observe, first of all, that the linear derivative term contains only the charged fields, i.e. $Q$ and $Q^{\dagger}$. The dispersion laws of the $P$ fields remain unaffected by the linear term. It remains Lorentz invariant, with mass given by the curvature of the static part of the Lagrangian. In order to determine the dispersion laws for the $Q$ and $Q^{\dagger}$ fields we need to solve a secular equation obtained by substituting Fourier decomposition of $Q$ 's into

$$
\begin{aligned}
\mathcal{L}(\Sigma)= & \mathcal{L}\left(\Sigma_{\alpha}\right)+\operatorname{Tr}\left\{\left(\partial_{\nu} Q_{R}^{\dagger} \partial_{\nu} Q_{R}+\partial_{\nu} Q_{I}^{\dagger} \partial_{\nu} Q_{I}\right)\right. \\
& \left.-4 \mu \cos \alpha\left(Q_{I}^{\dagger} \partial_{0} Q_{R}+Q_{R}^{\dagger} \partial_{0} Q_{I}\right)+4 \mu^{2} Q_{R}^{\dagger} Q_{R} \sin ^{2} \alpha\right\}+\ldots
\end{aligned}
$$

where we only wrote the $Q$-dependent terms. This expression is the same for either $\beta=1$ or $\beta=4$; only the definition of $Q$ 's is different: eqs. (70) or eqs. (75). The secular equation has the form

$$
\operatorname{det}\left(\begin{array}{cc}
E^{2}-\boldsymbol{p}^{2} & -4 \mu E \cos \alpha \\
-4 \mu E \cos \alpha & E^{2}-\boldsymbol{p}^{2}-4 \mu^{2} \sin ^{2} \alpha
\end{array}\right)=0 .
$$

\footnotetext{
${ }^{5}$ Note that though the mass of the diquarks $Q$ vanishes at the transition point, $\mu=m_{\pi} / 2$, the dispersion law is not linear, but quadratic, $E \sim \boldsymbol{p}^{2}$. This is related to the well-known critical slowing down at a second order phase transition.

${ }^{6}$ A useful observation which helps to write this in such a compact form is that $\operatorname{Tr}\left[X_{1}, \Pi\right] \partial_{0} \Pi=0$ for any $\Pi$. This can be checked explicitly using the block decomposition of $\Pi$, but it is easier to see that once you realize that the commutator of two $X$-like generators is a $T$-like generator, and its trace with another $X$-like generator, such as $\partial_{0} \Pi$ is zero.
} 
We see that $Q_{R}$ and $Q_{I}$ are mixed by the linear derivative term. The mixing is maximal when $\alpha=0$, and vanishes when $\alpha=\pi / 2$, i.e. when $\mu \gg m_{\pi}$. For any $\alpha$ there is a solution for which the rest energy $E(0)=0$ - the Goldstone boson branch, which we denote $\tilde{Q}$. For $\alpha=0$ it is given by $\tilde{Q}=Q=Q_{R}+Q_{I}$ (at rest, $\boldsymbol{p}=0$ ). For $\alpha=\pi / 2$ it is entirely $Q_{I}$. The other solution of the secular equation, the linear combination of $Q_{R}$ and $Q_{I}$ orthogonal to $\tilde{Q}$, is massive. We denote it by $\tilde{Q}^{\dagger}$.

The dispersion laws for the meson multiplets $P_{S}, P_{A}$ and the mixed diquark-antidiquark multiplets $\tilde{Q}$ and $\tilde{Q}^{\dagger}$ are given by (in the $\beta=1$ case) the following relations:

$$
\begin{aligned}
P_{S}: & E^{2}=\boldsymbol{p}^{2}+m_{\pi}^{2} x^{2} \\
P_{A} & : \quad E^{2}=\boldsymbol{p}^{2}+m_{\pi}^{2} x^{2} \cos ^{2} \alpha \\
\tilde{Q}^{\dagger}: & E^{2}=\boldsymbol{p}^{2}+2 \mu^{2}\left(1+3 \cos ^{2} \alpha\right)+2 \mu \sqrt{\mu^{2}\left(1+3 \cos ^{2} \alpha\right)^{2}+4 \boldsymbol{p}^{2} \cos ^{2} \alpha} \\
\tilde{Q} & : \quad E^{2}=\boldsymbol{p}^{2}+2 \mu^{2}\left(1+3 \cos ^{2} \alpha\right)-2 \mu \sqrt{\mu^{2}\left(1+3 \cos ^{2} \alpha\right)^{2}+4 \boldsymbol{p}^{2} \cos ^{2} \alpha} \quad \quad \quad(\beta=1)(86)
\end{aligned}
$$

The only difference in the dispersion laws for $\beta=4$ is the interchange of $P_{S}$ and $P_{A}$, and also that the degeneracy of the multiplets as given by (72) and (77) is different. Note that the dispersion relation for the lowest lying mode, i.e. $Q$, is linear: $E \sim p$. The slope is a function of $\mu$ : it vanishes at the transition point, $x=1$, and approaches 1 for large $\mu$. The linear slope of low energy excitations is characteristic of superfluidity.

The pole masses of the excitations, i.e. the position of the pole at zero momentum, $E(0)$, are given in Table 1 for $\beta=1$ and Table 2 for $\beta=4$. The representations of the residual symmetry groups are denoted by their Young diagrams. Because mesons are quark-quark or quark-antiquark pairs they transform as rank two tensors (unless they are singlets). The representations can be uniquely identified by their dimensions found in (17), (72) and (20), (77). An explicit form of the representations is given in the Appendix. Figures 1 and 2 show schematic pictures these results, together with the residual symmetry groups in the different phases. In particular, one observes that the spectrum is continuous at the transition point $x=1$.

\section{Diquark source}

In three-color QCD the diquark condensate is not a color-singlet, therefore, one cannot study the phenomenon of diquark condensation by applying an external diquark source: such a source term will not be gauge invariant. In the theories which we study in this paper, e.g., in two-color QCD, the diquark condensate is colorless. Therefore one can add a gauge-invariant source $j$ to the theory. This source plays a role similar to the role the quark mass $m$ plays with respect to the chiral condensate. Such a non-zero source term $j$ is in fact used in lattice simulations [5] in order to measure the value of the diquark condensate in the limit $j \rightarrow 0$. In this section we show how the results of the previous sections are modified in the presence of a non-zero $j$. 


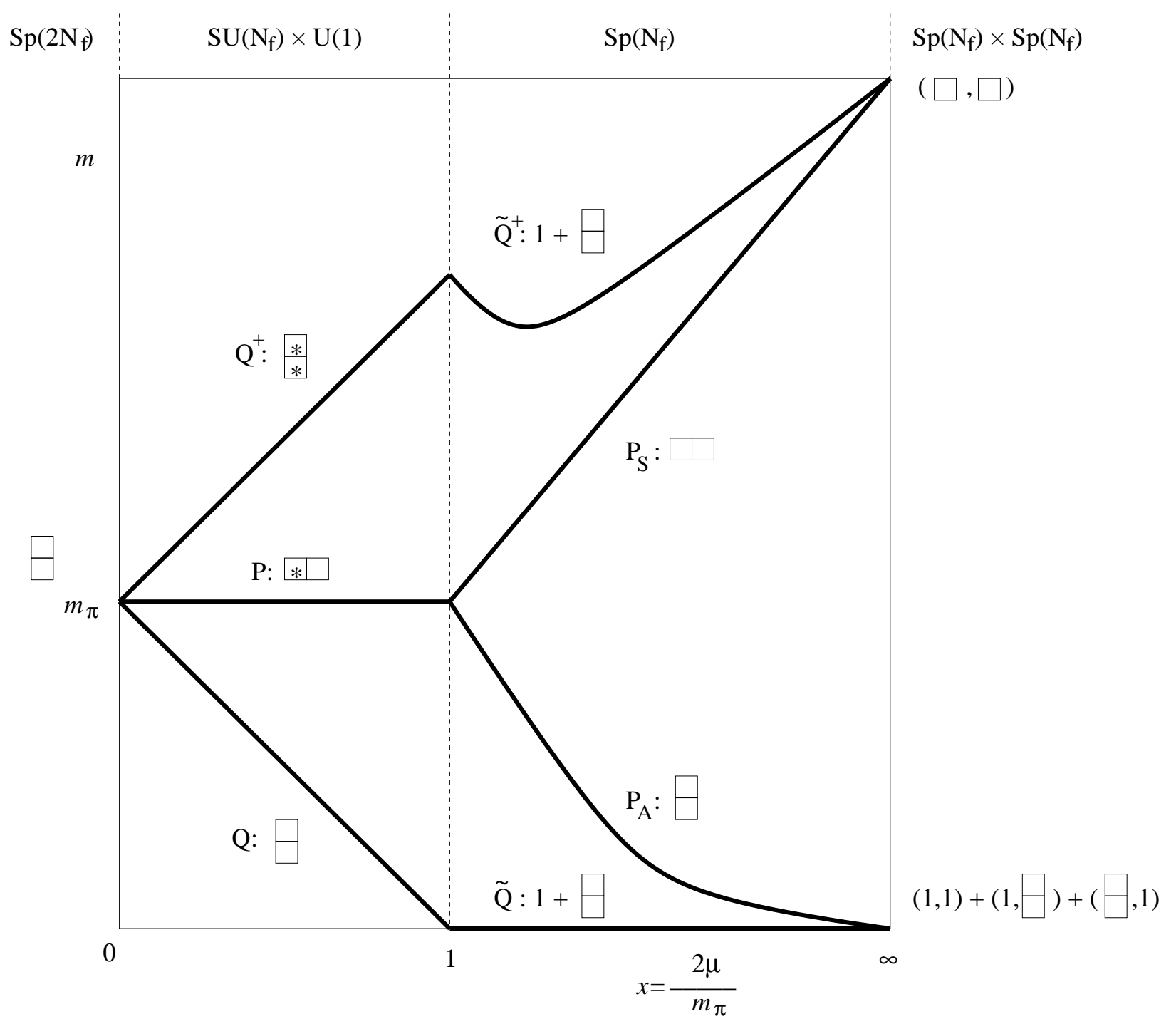

Figure 1: Spectrum of two-color QCD $(\beta=1)$ at finite $\mu$ and $m$ (schematic). The branches are labeled according to (81), (70) and 75) and by Young's diagrams showing the multiplet structure under the corresponding residual symmetry. The star denotes the conjugate fundamental representation. The residual symmetry groups are marked above the plot. The degeneracies are given by (17) and (72). For example, for the case of $N_{f}=2$ we find $N_{P}=3$, $N_{Q}=N_{Q^{\dagger}}=1$, and $N_{P_{S}}=3$. The branch $P_{A}$ does not exist for $N_{f}=2$. On the left $/$ right of the plot the residual symmetries and multiplet structure of the corresponding limiting cases $\mu=0$ and $m=0$ are shown. 
Table 1: Spectrum (masses and degeneracies) of two-color QCD with fundamental quarks $(\beta=1)$ :

\begin{tabular}{|c|c|c|c|c|c|c|}
\hline & \multicolumn{3}{|c|}{$\mu<m_{\pi} / 2: S U\left(N_{f}\right) \times U(1)$} & \multicolumn{3}{|c|}{$\mu>m_{\pi} / 2: S p\left(N_{f}\right)$} \\
\hline & mass & degen. & rep. & mass & degen. & rep. \\
\hline$P_{S}$ & \multirow[t]{2}{*}{$m_{\pi}$} & \multirow[t]{2}{*}{$N_{f}^{2}-1$} & \multirow[t]{2}{*}{\begin{tabular}{|l|l}
$*$ & \\
\end{tabular}} & $2 \mu$ & $\frac{1}{2} N_{f}\left(N_{f}+1\right)$ & \\
\hline$P_{A}$ & & & & $m_{\pi}^{2} / 2 \mu$ & $\frac{1}{2} N_{f}\left(N_{f}-1\right)-1$ & \\
\hline$Q^{\dagger}$ & $m_{\pi}+2 \mu$ & $\frac{1}{2} N_{f}\left(N_{f}-1\right)$ & \begin{tabular}{|l|}
$*$ \\
$*$ \\
\end{tabular} & $2 \mu \sqrt{1+3\left(m_{\pi} / 2 \mu\right)^{4}}$ & $\frac{1}{2} N_{f}\left(N_{f}-1\right)$ & $1+$ \\
\hline$Q$ & $m_{\pi}-2 \mu$ & $\frac{1}{2} N_{f}\left(N_{f}-1\right)$ & 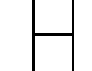 & 0 & $\frac{1}{2} N_{f}\left(N_{f}-1\right)$ & $1+$ \\
\hline
\end{tabular}

Table 2: Spectrum (masses and degeneracies) of any-color QCD with adjoint quarks $(\beta=4)$.

\begin{tabular}{|c|c|c|c|c|c|c|}
\hline & \multicolumn{3}{|c|}{$\mu<m_{\pi} / 2: S U\left(N_{f}\right) \times U(1)$} & \multicolumn{3}{|c|}{$\mu>m_{\pi} / 2: S O\left(N_{f}\right)$} \\
\hline & mass & degen. & rep. & mass & degen. & rep. \\
\hline$P_{A}$ & $m_{\pi}$ & $N_{f}^{2}-1$ & \begin{tabular}{|l|l}
$*$ & \\
\end{tabular} & $2 \mu$ & $\frac{1}{2} N_{f}\left(N_{f}-1\right)$ & \\
\hline$P_{S}$ & & & & $m_{\pi}^{2} / 2 \mu$ & $\frac{1}{2} N_{f}\left(N_{f}+1\right)-1$ & \\
\hline$Q^{\dagger}$ & $m_{\pi}+2 \mu$ & $\frac{1}{2} N_{f}\left(N_{f}+1\right)$ & \begin{tabular}{|l|l|}
$*$ & $*$ \\
\end{tabular} & $2 \mu \sqrt{1+3\left(m_{\pi} / 2 \mu\right)^{4}}$ & $\frac{1}{2} N_{f}\left(N_{f}+1\right)$ & $1+[$ \\
\hline$Q$ & $m_{\pi}-2 \mu$ & $\frac{1}{2} N_{f}\left(N_{f}+1\right)$ & \begin{tabular}{|l|l|}
$\square$ \\
\end{tabular} & 0 & $\frac{1}{2} N_{f}\left(N_{f}+1\right)$ & $1+[$ \\
\hline
\end{tabular}




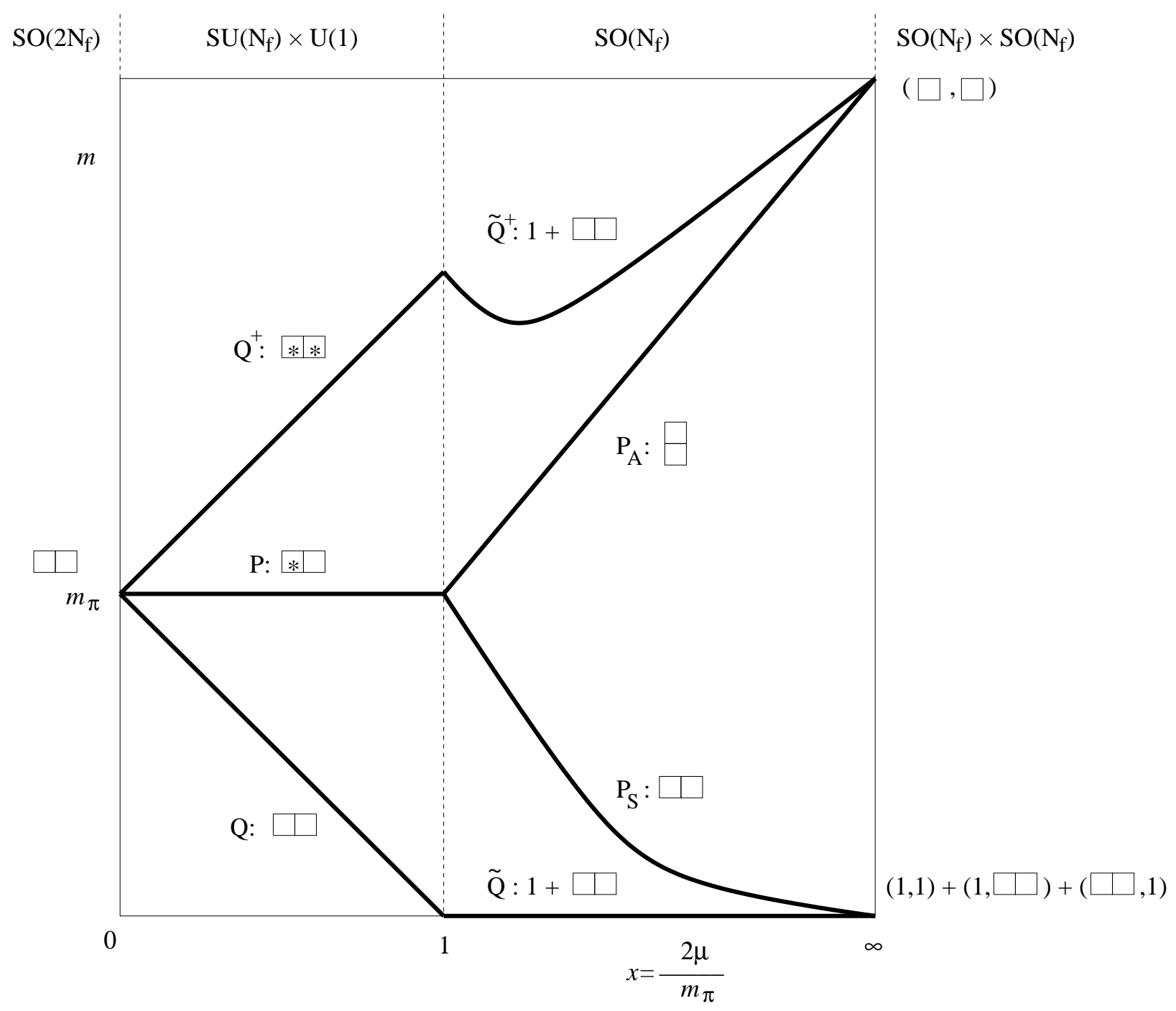

Figure 2: Spectrum of any-color QCD with adjoint quarks $(\beta=4)$ at finite $\mu$ and $m$ (schematic). The branches are labeled according to (81), (70) and (75) and by Young's diagrams showing the multiplet structure under the corresponding residual symmetry. The residual symmetry groups are marked above the plot. The degeneracies are given in Table 2. For example, for the case of $N_{f}=2: N_{P}=3, N_{Q}=6, N_{Q}=N_{Q^{\dagger}}=3, N_{P_{S}}=2$ and $N_{P_{A}}=1$. On the left/right of the plot the residual symmetries and multiplet structure of the corresponding limiting cases $\mu=0$ and $m=0$ are shown. 


\section{$11.1 \beta=1$}

Let us first rewrite the scalar diquark source term in the macroscopic theory using our $S U\left(2 N_{f}\right)$ spinor notations. In the notations of section of we find

$$
\begin{array}{cc}
-i \frac{j}{2} \psi^{T} C \gamma_{5} \tau_{2} I \psi+\text { h.c. }=-\frac{j}{2} \Psi^{T} \sigma_{2} \tau_{2}\left(\begin{array}{cc}
i I & 0 \\
0 & i I
\end{array}\right) \Psi+\text { h.c. } \equiv-\frac{1}{2} \Psi^{T} \sigma_{2} \tau_{2} J \Psi+\text { h.c. }, \\
J=j \hat{J} \quad \text { and } \quad \hat{J}=\left(\begin{array}{cc}
i I & 0 \\
0 & i I
\end{array}\right), & (\beta=1)
\end{array}
$$

the antisymmetric matrix $I$ is defined in (45), and the summation over the $N_{f}$ flavor indices has been suppressed. Comparing with the quark mass term (22) we find that the two belong to the same multiplet (i.e. they transform into one another) under the $S U\left(2 N_{f}\right)$ rotations.7 We can write the sum of the diquark source and the bare mass term as

$$
m \bar{\psi} \psi-i \frac{j}{2}\left(\psi^{T} C \gamma_{5} \tau_{2} I \psi+\text { h.c. }\right)=-\frac{1}{2} \Psi^{T} \sigma_{2} \tau_{2} M_{\phi} \Psi, \quad(\beta=1)
$$

where

$$
M_{\phi}=m \hat{M}+j \hat{J}=\sqrt{m^{2}+j^{2}}(\hat{M} \cos \phi+\hat{J} \sin \phi)=\sqrt{m^{2}+j^{2}} \hat{M}_{\phi} .
$$

We have used a notation similar to (47) with

$$
\tan \phi=\frac{j}{m}
$$

It is easy to see how the diquark source term modifies the effective Lagrangian (42): we need to replace the mass matrix $m G \hat{M}$ in (29) by $\sqrt{m^{2}+j^{2}} G \hat{M}_{\phi}$. The Gell-Mann-Oakes-Renner relation at $\mu=0$ becomes

$$
m_{\pi}^{2}=\frac{\sqrt{m^{2}+j^{2}} G}{F^{2}}
$$

Using this fact we can write the effective Lagrangian for the theory with both mass term and diquark source (compare to (42)) as

$$
\mathcal{L}_{\text {eff }}(\Sigma)=\frac{F^{2}}{2}\left[\operatorname{Tr} \nabla_{\nu} \Sigma \nabla_{\nu} \Sigma^{\dagger}-2 m_{\pi}^{2} \operatorname{Re} \operatorname{Tr}\left(\hat{M}_{\phi} \Sigma\right)\right]
$$

Now we can repeat the steps we performed in section 9, but for the Lagrangian (92).

Since the source term we introduced favors the direction of $\Sigma_{d}$, we expect that the minimum of the Lagrangian (92), $\bar{\Sigma}$, is again given by a linear combination of $\Sigma_{c}$ and $\Sigma_{d}$ as in (47),

$$
\bar{\Sigma}=\Sigma_{\alpha}=\Sigma_{c} \cos \alpha+\Sigma_{d} \sin \alpha=V_{\alpha} \Sigma_{c} V_{\alpha}^{T}, \quad \text { where } \quad V_{\alpha}=e^{i \alpha X_{2}},
$$

${ }^{7}$ There is a freedom in the choice of the orientation of the diquark source $\hat{J}$. It is precisely the same freedom as the one corresponding to the $S U\left(N_{f}\right) \times U(1) / S p\left(N_{f}\right)$ degeneracy of the diquark condensation vacuum $\Sigma_{d}$. An infinitesimal diquark source $j$ determines the vacuum orientation of $\Sigma_{d}$. Our choices reflect this fact. Indeed $\Sigma_{d}=\hat{J}^{\dagger}$. 
and $X_{2}$ is the generator that rotates $\Sigma_{c}$ into $\Sigma_{d}$ as before. The value of $\alpha$, as determined by the saddle-point equations, now depends on the value of the diquark source. The proof of Section 8 is not easily extendable to this case. We shall continue on the assumption that the minimum is global. We shall prove, however, that it is a minimum when we expand to second order in fluctuations of $\Sigma$ and find no linear terms and a positive quadratic form. If we substitute this ansatz into the effective Lagrangian (92) we find

$$
\mathcal{L}_{\mathrm{st}}\left(\Sigma_{\alpha}\right)=F^{2} m_{\pi}^{2} N_{f}\left[\frac{x^{2}}{2}(\cos 2 \alpha-1)-2 \cos (\alpha-\phi)\right] .
$$

The dependence of the angle $\alpha$ on $x$ follows from minimizing this Lagrangian and is given by

$$
x^{2} \cos \alpha \sin \alpha=\sin (\alpha-\phi),
$$

which is different from (49), but coincides with it in the limit $\phi \rightarrow 0$, of course. For finite $\phi$ the angle $\alpha$ is already non-zero $(\alpha=\phi)$ at $x=0$ as it should be, since a diquark source drives a non-zero diquark condensate. At $x=\infty$, the value of $\alpha$ is $\pi / 2$ independently of $\phi$. The major difference from (49) is that the dependence of $\alpha$ on $x$ is analytic. There is no phase transition as a function of $\mu$ when the diquark source $j$ is nonzero. This is to be expected: the diquark source plays the role an external magnetic field plays in a ferromagnet. The diquark source explicitly breaks $S U\left(N_{f}\right)_{V} \times U(1)_{B}$ symmetry down to $S p\left(N_{f}\right)_{V}$ and there is only one phase (the one with residual $S p\left(N_{f}\right)_{V}$ symmetry) for all values of $x$.

Our next step is to expand in powers of fluctuations of $\Sigma$ around $\bar{\Sigma}$. This can be done following the algebra in section 9 with minimal modifications. The Goldstone manifold is again parameterized by

$$
\Sigma=V_{\alpha} U \Sigma_{c} U^{T} V_{\alpha}^{T},
$$

but the second identity in (64) is now given by

$$
V_{\alpha}^{T} \hat{M}_{\phi} V_{\alpha}=\left(V_{-\alpha} \hat{M}_{\phi}^{\dagger} V_{-\alpha}^{T}\right)^{\dagger}=\Sigma_{\phi-\alpha}^{\dagger}=\Sigma_{c}^{\dagger} \cos (\alpha-\phi)-\Sigma_{d}^{\dagger} \sin (\alpha-\phi),
$$

whereas the first identity is the same as before. Using this algebra, the effective Lagrangian to second order in the pion fields can be expressed as

$$
\begin{aligned}
\mathcal{L}(\Sigma) & =\mathcal{L}_{\mathrm{st}}\left(\Sigma_{\alpha}\right)+F m_{\pi}^{2}\left[x^{2} \cos \alpha \sin \alpha-\sin (\alpha-\phi)\right] \operatorname{Tr}\left(X_{2} \Pi\right) \\
& +\frac{1}{2} \operatorname{Tr}\left\{\left(\partial_{\nu} \Pi\right)^{2}-2 \mu \cos \alpha[B, \Pi] \partial_{0} \Pi\right\} \\
& +\frac{m_{\pi}^{2}}{2}\left[\frac{x^{2}}{4}\left(\operatorname{Tr}[B, \Pi]^{2} \cos ^{2} \alpha-\operatorname{Tr}\left[X_{1}, \Pi\right]^{2} \sin ^{2} \alpha\right)+\operatorname{Tr} \Pi^{2} \cos (\alpha-\phi)\right]+\ldots
\end{aligned}
$$

Inspecting the linear terms we find that they vanish if $\alpha$ is chosen according to (95). This means that our ansatz (93) is indeed an extremum.

In order to find the mass spectrum we have to study the term of second order in the $\Pi$-fields in the Lagrangian (98), and determine the dispersion laws of the different (pseudo)Goldstone fields. Using block decomposition of the generators $\Pi$ (16), projections (70) of 
$P$ and $Q$ and the relationship (95) between $x$ and $\alpha$ we find

$$
\begin{aligned}
\mathcal{L}(\Sigma)= & \mathcal{L}_{\mathrm{st}}\left(\Sigma_{\alpha}\right)+\operatorname{Tr}\left[\left(\partial_{\nu} Q_{R}^{\dagger} \partial_{\nu} Q_{R}+\partial_{\nu} Q_{I}^{\dagger} \partial_{\nu} Q_{I}\right)-4 \mu \cos \alpha\left(Q_{I}^{\dagger} \partial_{0} Q_{R}+Q_{R}^{\dagger} \partial_{0} Q_{I}\right)\right] \\
& +m_{\pi}^{2} \operatorname{Tr}\left[Q_{I} Q_{I}^{\dagger} \frac{\sin \phi}{\sin \alpha}+Q_{R} Q_{R}^{\dagger}\left(x^{2} \sin ^{2} \alpha+\frac{\sin \phi}{\sin \alpha}\right)\right] \\
& +\operatorname{Tr}\left[\partial_{\nu} P_{A} \partial_{\nu} P_{A}+P_{A}^{2} m_{\pi}^{2}\left(x^{2} \cos ^{2} \alpha+\frac{\sin \phi}{\sin \alpha}\right)\right] \\
& +\operatorname{Tr}\left[\partial_{\nu} P_{S} \partial_{\nu} P_{S}+P_{S}^{2} m_{\pi}^{2}\left(x^{2}+\frac{\sin \phi}{\sin \alpha}\right)\right]+\ldots \quad(\beta=1)
\end{aligned}
$$

As in the case of a vanishing diquark source studied in the previous Section, the linear derivative term contains only the charged fields $Q$ and $Q^{\dagger}$. Therefore the dispersion relations for the neutral fields $P$ are not affected by this term: the masses of the $P$ fields are given by the curvature of the static part of the Lagrangian at the minimum. However the linear derivative term mixes the charged Goldstones. In order to obtain the dispersion laws for the $Q$ fields, as in Section 10, we have to solve a secular equation obtained by substituting the Fourier decomposition of these fields into the effective Lagrangian (99). The secular equation reads

$$
\operatorname{det}\left(\begin{array}{cc}
E^{2}-\boldsymbol{p}^{2}-m_{\pi}^{2} \frac{\sin \phi}{\sin \alpha} & -4 \mu E \cos \alpha \\
-4 \mu E \cos \alpha & E^{2}-\boldsymbol{p}^{2}-4 \mu^{2} \sin ^{2} \alpha-m_{\pi}^{2} \frac{\sin \phi}{\sin \alpha}
\end{array}\right)=0 .
$$

The dispersion laws for the different Goldstone modes are therefore found to be given by

$$
\begin{gathered}
P_{S}: \quad E^{2}=\boldsymbol{p}^{2}+m_{\pi}^{2}\left(x^{2}+\frac{\sin \phi}{\sin \alpha}\right) ; \\
P_{A}: E^{2}=\boldsymbol{p}^{2}+m_{\pi}^{2}\left(x^{2} \cos ^{2} \alpha+\frac{\sin \phi}{\sin \alpha}\right) ; \\
\tilde{Q}^{\dagger}: \quad E^{2}=\boldsymbol{p}^{2}+m_{\pi}^{2} \frac{\sin \phi}{\sin \alpha}+2 \mu^{2}\left(1+3 \cos ^{2} \alpha\right) \\
\quad+2 \mu \sqrt{\mu^{2}\left(1+3 \cos ^{2} \alpha\right)^{2}+4 \cos ^{2} \alpha\left(\boldsymbol{p}^{2}+m_{\pi}^{2} \frac{\sin \phi}{\sin \alpha}\right)} ; \\
\tilde{Q}: \quad E^{2}=\boldsymbol{p}^{2}+m_{\pi}^{2} \frac{\sin \phi}{\sin \alpha}+2 \mu^{2}\left(1+3 \cos ^{2} \alpha\right) \\
\quad-2 \mu \sqrt{\mu^{2}\left(1+3 \cos ^{2} \alpha\right)^{2}+4 \cos ^{2} \alpha\left(\boldsymbol{p}^{2}+m_{\pi}^{2} \frac{\sin \phi}{\sin \alpha}\right) ; \quad(\beta=1),}
\end{gathered}
$$

where $\alpha$ is an implicit function of $x$ and $\phi$ given by (95)). Note that the $\tilde{Q}$ are no longer true Goldstone modes, since the symmetry, which is broken spontaneously in the diquark condensation phase $x>1$ at $j=0$, is now broken explicitly by the diquark source term. The masses of the different multiplets are given by the value of $E$ at $\boldsymbol{p}=0$. The positivity of all masses shows that the minimum given by the saddle-point equation (95) is at least a local minimum. This mass spectrum at a small non-zero diquark source is depicted in Fig. 斿. 


\section{$11.2 \beta=4$}

In the $\beta=4$ case the diquark source term has the form

$$
\begin{gathered}
i \frac{j}{2} \psi^{T} C \gamma_{5} \psi+\text { h.c. }=\frac{j}{2} \Psi^{T} \sigma_{2}\left(\begin{array}{cc}
i & 0 \\
0 & i
\end{array}\right) \Psi+\text { h.c. } \equiv-\frac{1}{2} \Psi^{T} \sigma_{2} J \Psi+\text { h.c. } \\
J=j \hat{J} \quad \text { and } \quad \hat{J}=-\left(\begin{array}{cc}
i & 0 \\
0 & i
\end{array}\right) \quad(\beta=4)
\end{gathered}
$$

The analysis follows the same lines as in the $\beta=1$ case. We again introduce the combined mass matrix

$$
M_{\phi}=m \hat{M}+j \hat{J}=\sqrt{m^{2}+j^{2}} \hat{M}_{\phi},
$$

with $\hat{M}$ and $\hat{J}$ defined for $\beta=4$ in (33) and (102). In the expansion of the Lagrangian to second order in the $\Pi$-fields for $\beta=1$ we have only used the general commutation properties of $\hat{M}_{\phi}$ which, with a proper redefinition of the generators $X_{1}$ and $X_{2}$, are the same in the case $\beta=4$. We thus obtain the same second order Lagrangian (98) as for $\beta=1$ resulting in the same saddle point equation (95) and the same mean field value of the free energy. However, the mass spectrum is slightly different. We express the quadratic part of the Lagrangian in terms of the block-decomposition (16) and the projections (75). The final result is

$$
\begin{aligned}
\mathcal{L}(\Sigma)= & \mathcal{L}_{\text {st }}\left(\Sigma_{\alpha}\right)+\operatorname{Tr}\left[\left(\partial_{\nu} Q_{R}^{\dagger} \partial_{\nu} Q_{R}+\partial_{\nu} Q_{I}^{\dagger} \partial_{\nu} Q_{I}\right)-4 \mu \cos \alpha\left(Q_{I}^{\dagger} \partial_{0} Q_{R}+Q_{R}^{\dagger} \partial_{0} Q_{I}\right)\right] \\
& +m_{\pi}^{2} \operatorname{Tr}\left[Q_{I} Q_{I}^{\dagger} \frac{\sin \phi}{\sin \alpha}+Q_{R} Q_{R}^{\dagger}\left(x^{2} \sin ^{2} \alpha+\frac{\sin \phi}{\sin \alpha}\right)\right] \\
& +\operatorname{Tr}\left[\partial_{\nu} P_{S} \partial_{\nu} P_{S}+P_{S}^{2} m_{\pi}^{2}\left(x^{2} \cos ^{2} \alpha+\frac{\sin \phi}{\sin \alpha}\right)\right] \\
& +\operatorname{Tr}\left[\partial_{\nu} P_{A} \partial_{\nu} P_{A}+P_{A}^{2} m_{\pi}^{2}\left(x^{2}+\frac{\sin \phi}{\sin \alpha}\right)\right]+\ldots \quad(\beta=4)
\end{aligned}
$$

As in the $\beta=1$ case, the linear derivative term mixes the $Q$ and $Q^{\dagger}$ fields. In order to get the dispersion relations of these fields, the same secular equation as before (100) has to be solved. The dispersion laws are the same as in (101) with the familiar exchange of labels $S \leftrightarrow A$, and the degeneracy of the multiplets given by (77) instead of (72). The corresponding mass spectrum at a small non-zero $j$ is shown in Fig. 3 .

\section{Condensate and baryon charge densities}

In this section we derive the dependence of the chiral condensate, the diquark condensate and the baryon charge densities. These densities are obtained by differentiating the vacuum energy density with respect to $m, j$ and $\mu$,

$$
\langle\bar{\psi} \psi\rangle=-\frac{\partial \mathcal{E}_{\mathrm{vac}}}{\partial m} ; \quad\langle\psi \psi\rangle=-\frac{\partial \mathcal{E}_{\mathrm{vac}}}{\partial j} ; \quad n_{B}=-\frac{\partial \mathcal{E}_{\mathrm{vac}}}{\partial \mu} .
$$

\footnotetext{
${ }^{8}$ As before, our previous choice of $\Sigma_{d}$ corresponds to our choice of $J$, i.e., $\Sigma_{d}=\hat{J}^{\dagger}$.
} 


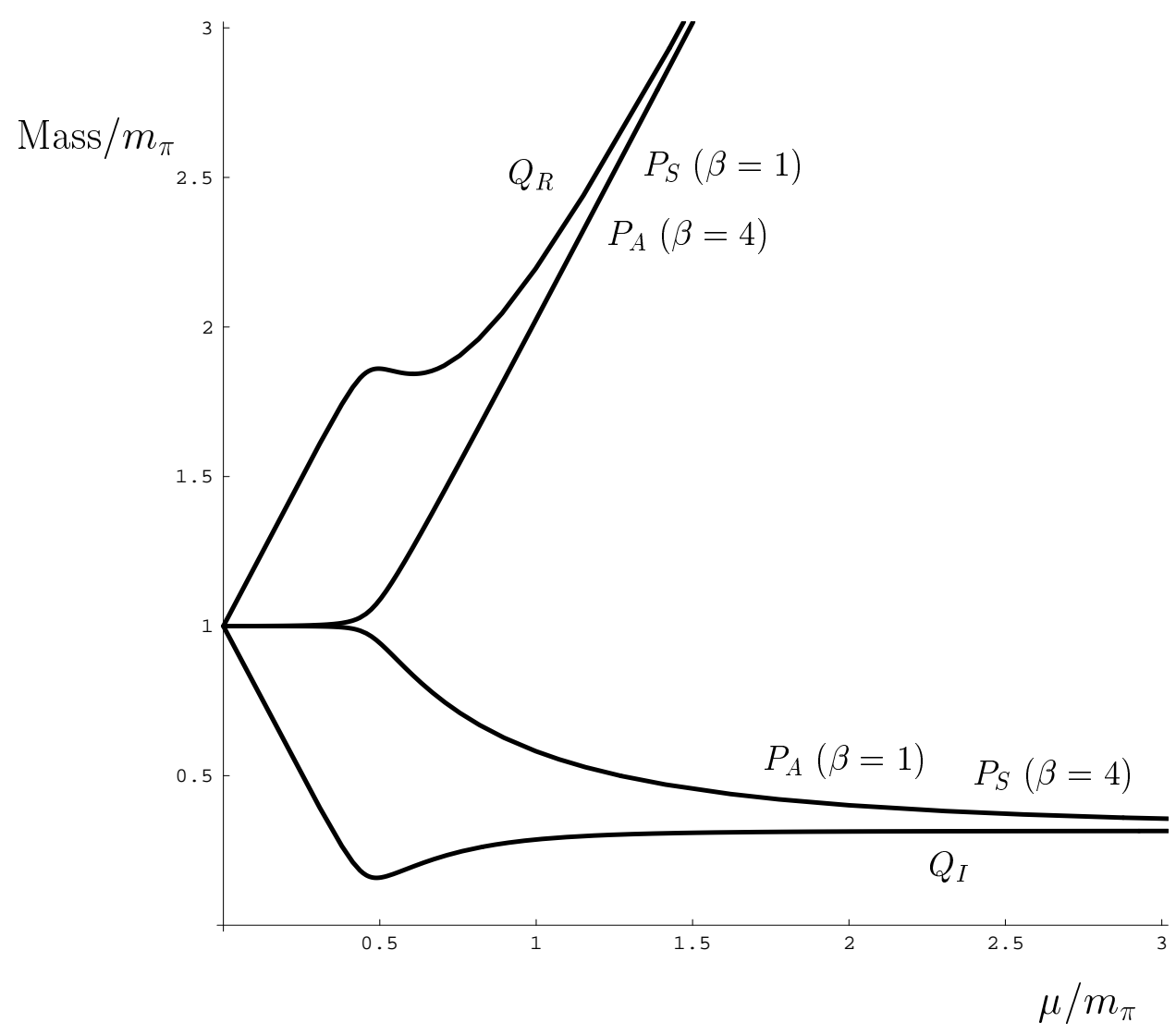

Figure 3: Mass spectrum for a small non-vanishing source $j=0.1 \mathrm{~m}$. 
The shorthand symbol $\psi \psi$ denotes the l.h.s. of the equation (87) in the $\beta=1$ case and of (102) in the $\beta=4$ case without factor $j$.

In the effective theory the vacuum energy density is given by the value of the effective Lagrangian (42) at the minimum,

$$
\begin{aligned}
\mathcal{E}_{\mathrm{vac}}=\mathcal{L}_{\mathrm{eff}}\left(\Sigma_{\alpha}\right)= & \frac{F^{2}}{2}\left[-2 \mu^{2} \operatorname{Tr}\left(\Sigma_{\alpha} B^{T} \Sigma_{\alpha}^{\dagger} B+B B\right)-2 m_{\pi}^{2} \operatorname{Re} \operatorname{Tr}\left(\Sigma_{\phi}^{\dagger} \Sigma_{\alpha}\right)\right] \\
& =-4 N_{f} F^{2} \mu^{2} \sin ^{2} \alpha-2 N_{f} G(m \cos \alpha+j \sin \alpha),
\end{aligned}
$$

where we used (90) and (91). Differentiating'g, we find

$$
\langle\bar{\psi} \psi\rangle=2 N_{f} G \cos \alpha ; \quad\langle\psi \psi\rangle=2 N_{f} G \sin \alpha ; \quad n_{B}=8 N_{f} F^{2} \mu \sin ^{2} \alpha,
$$

where the angle of the rotation of the condensate, $\alpha$, depends implicitly on $\mu, m$ and $j$ through the solution of (95). Note, in particular, that the sum $\langle\bar{\psi} \psi\rangle^{2}+\langle\psi \psi\rangle^{2}$ does not depend on $\mu, m$ or $j$ (to the order in Chiral Perturbation Theory we are working), which reflects the fact that the condensate rotates.

For $j=0$ the dependence of $\alpha$ on $\mu$ is simple: $\alpha=0$, for $\mu<m_{\pi} / 2$, and $\alpha=$ $\arccos \left[m_{\pi}^{2} /\left(4 \mu^{2}\right)\right]$ otherwise (49). That means the densities (107) are constant for $\mu<m_{\pi} / 2$, as they should since the vacuum state does not change until $\mu$ reaches $m_{\pi} / 2$. The results for the condensate and baryon charge densities are summarized in Table 3.

Table 3: The values of the chiral condensate, $\langle\bar{\psi} \psi\rangle$, the diquark condensate, $\langle\psi \psi\rangle$, and the baryon density $n_{B}$ in the two phases of the theory.

\begin{tabular}{|c||c|c|c|}
\hline phase & $\langle\bar{\psi} \psi\rangle$ & $\langle\psi \psi\rangle$ & $n_{B}$ \\
\hline$\mu<m_{\pi} / 2$ & $\langle\bar{\psi} \psi\rangle_{0}$ & 0 & 0 \\
$\mu>m_{\pi} / 2$ & $\langle\bar{\psi} \psi\rangle_{0}\left(\frac{m_{\pi}}{2 \mu}\right)^{2}$ & $\langle\bar{\psi} \psi\rangle_{0} \sqrt{1-\left(\frac{m_{\pi}}{2 \mu}\right)^{4}}$ & $8 \mu N_{f} F^{2}\left(1-\left(\frac{m_{\pi}}{2 \mu}\right)^{4}\right)$ \\
\hline
\end{tabular}

For $j \neq 0$ the angle $\alpha$ varies between the values $\phi$ and $\pi / 2$ as a function of $\mu$ and $m$, determined by the saddle point equation (95) $x^{2} \sin \alpha \cos \alpha=\sin (\alpha-\phi)$. The saddle point is a quartic equation which, in principle, can be solved analytically for arbitrary $j$. The dependence of the densities (107) on $\mu$ is shown in Fig. 1 for $j=0$ and in Fig. 5 for $j \neq 0$.

\section{Equation of state of the non-ideal diquark gas}

We now take a different look at the system we study (quarks with two colors or any-color adjoint quarks) near the threshold, $\mu \approx m_{\pi} / 2$. First of all, we observe, that for $\mu$ very close to $m_{\pi} / 2$ the density of baryons is very (arbitrarily) small. This means that we can describe the system by an almost ideal gas. It should be a Bose gas, since the baryons, i.e. diquarks, are bosons. The equation of state of a dilute non-ideal Bose gas is a textbook problem

\footnotetext{
${ }^{9}$ The implicit dependence of $\alpha$ on $m, j$ and $\mu$ is not contributing because $\partial \mathcal{E}_{\mathrm{vac}} / \partial \alpha=0$.
} 


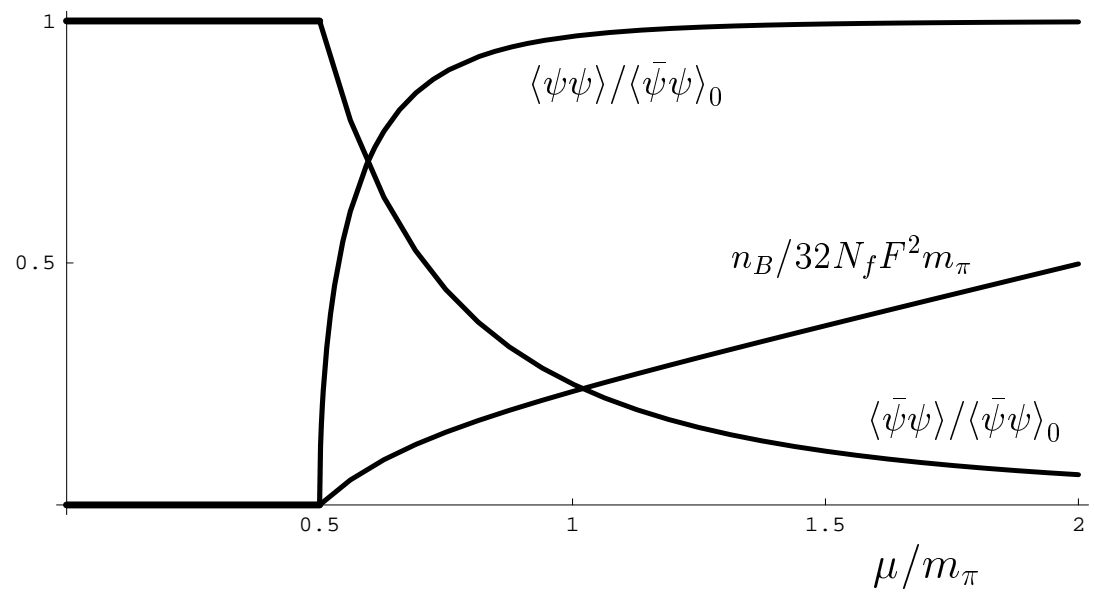

Figure 4: The magnitudes of the chiral $\langle\bar{\psi} \psi\rangle$ and the diquark $\langle\psi \psi\rangle$ condensates in units of $\langle\bar{\psi} \psi\rangle_{0}=2 N_{f} G$ as a function of $\mu / m_{\pi}$ for zero diquark source. Also the density of the baryon charge in units of $32 N_{f} F^{2} m_{\pi}$ is shown. 


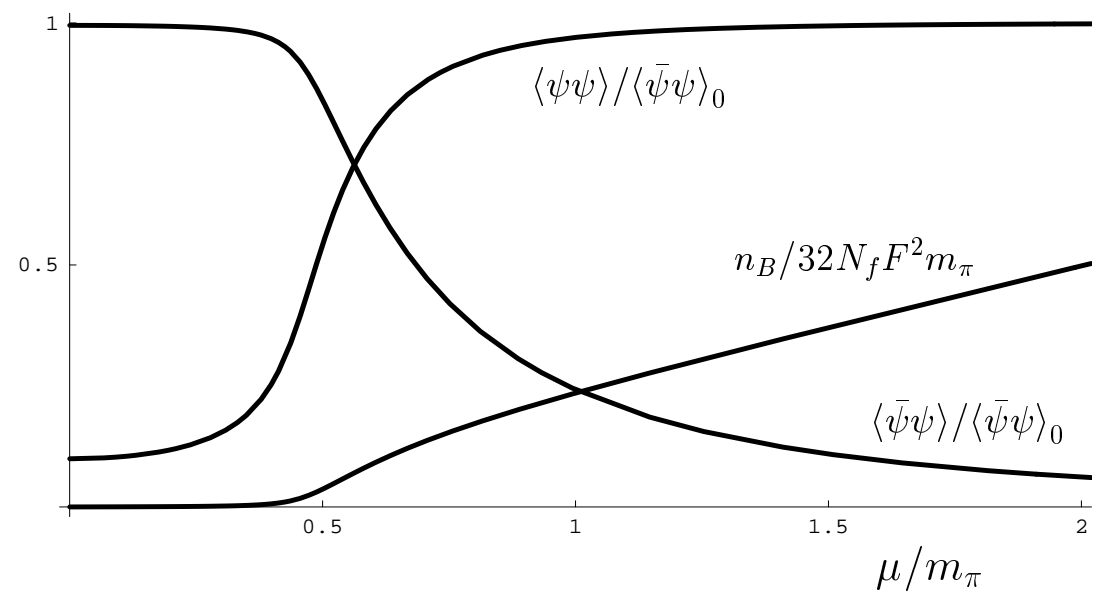

Figure 5: The magnitudes of the chiral $\langle\bar{\psi} \psi\rangle$ and the diquark $\langle\psi \psi\rangle$ condensates in units of $2 N_{f}\langle\bar{\psi} \psi\rangle_{0}$ as a function of $\mu / m_{\pi}$ at small non-zero diquark source $j=0.1 m$. Also the density of the baryon charge in units of $32 N_{f} F^{2} m_{\pi}$ is shown. 
[31]. The interaction between the bosons is crucial here (the dependence on its strength is non-analytic, e.g. the attractive Bose gas is unstable). The baryons in our system repel each other, as we shall see. Moreover, we shall also see that the strength of this repulsion is exactly the one that gives the correct equation of state (107), (49) at small densities:

$$
n_{B}=32 N_{f} F^{2}\left(\mu-\frac{m_{\pi}}{2}\right)+\ldots
$$

This is a very non-trivial check of the consistency of our effective Lagrangian!

Since we are working at very small densities, we can expand the Lagrangian (29) up to next (quartic order) in the Goldstone fields. The quartic terms will describe the interaction energy of the Goldstones due to two-body scattering,

$$
\mathcal{E}_{\text {int }}=-\frac{1}{24 F^{2}} \operatorname{Tr}\left[\Pi, \partial_{\mu} \Pi\right]\left[\Pi, \partial^{\mu} \Pi\right]-\frac{m_{\pi}^{2}}{24 F^{2}} \operatorname{Tr} \Pi^{4}+\ldots .
$$

We are now working in Minkowski space and the extra minus sign in front of the first term, compared to (29), is a consequence of this. The presence of the time derivatives should not confuse the reader. Since we are dealing with a non-relativistic system, $\mu-m_{\pi} / 2 \ll m_{\pi}$, we only need the leading term in the time dependence of the relativistic fields, i.e. $\partial_{0} \Pi \rightarrow$ $\pm i m_{\pi} \Pi$. The spatial derivatives give only a subleading contribution. The dominant effect is that of the $s$-wave amplitude.

We now consider the ground state of such a system with the baryon density fixed (unlike before, when we fixed $\mu$ ). Most of the particles will occupy the lowest energy, i.e., zeromomentum, level (the population of the excited levels is the next order effect). These particles form a condensate, whose amplitude we denote by $\Pi$. The energy of the system is different from zero only because of the interaction, and, as a function of $\Pi$, it is given to us by (109). Looking at the block decomposition of $\Pi(16)$ we note that only $Q$ and $Q^{\dagger}$ (diquark and antidiquark) fields carry non-zero baryon number. Therefore, we set $P=0$ in our case. Thus, from (109), we find for the dependence of the energy on the magnitude of the $Q$ condensate

$$
\mathcal{E}_{\text {int }}=\frac{m_{\pi}^{2}}{4 F^{2}} \operatorname{Tr}\left(Q Q^{\dagger}\right)^{2}+\ldots,
$$

where we neglected subleading non-relativistic corrections.

Now we need to calculate $n_{B}$ as a function of $Q$. Since $Q$ carries baryon charge 2, we can write

$$
n_{B}=2 \operatorname{Tr}\left(Q^{\dagger} i \partial_{0} Q-Q i \partial_{0} Q^{\dagger}\right)=4 m_{\pi} \operatorname{Tr} Q Q^{\dagger}+\ldots,
$$

where we neglected subleading corrections. Since all flavors of diquarks are equivalent $\square$ the matrix $Q$ is proportional to $I$ for $\beta=1$, or to 1 for $\beta=4$. Thus we can relate traces in

\footnotetext{
${ }^{10}$ Another way to see (111) is to use the fact that $n_{B}=-\partial \mathcal{L} / \partial \mu$. Thus, from (42) we find that $n_{B}=$ $2 F^{2} \operatorname{Tr} B \Sigma^{\dagger} \partial_{0} \Sigma=2 i \operatorname{Tr} B \Pi \partial_{0} \Pi$, which coincides with (111).

${ }^{11}$ There is only one flavor of diquarks for $\beta=1, \overline{N_{f}}=2$. For larger $N_{f}$ the flavor $S U\left(N_{f}\right)$ symmetry ensures the equivalence of all diquarks.
} 
(110) and (111) by $\operatorname{Tr}\left(Q Q^{\dagger}\right)^{2}=\left(\operatorname{Tr} Q Q^{\dagger}\right)^{2} / N_{f}$. Therefore, we find for the dependence on $n_{B}$ of the vacuum energy at fixed $n_{B}$,

$$
\mathcal{E}_{\mathrm{vac}}=\frac{n_{B}}{2} m_{\pi}+\mathcal{E}_{\mathrm{int}}=\frac{n_{B}}{2} m_{\pi}+\frac{n_{B}^{2}}{64 F^{2} N_{f}}+\ldots
$$

The first term is the rest energy of the diquarks. This determines the equation of state

$$
\mu \equiv \frac{\partial \mathcal{E}_{\mathrm{vac}}}{\partial n_{B}}=\frac{m_{\pi}}{2}+\frac{n_{B}}{32 F^{2} N_{f}}+\ldots
$$

The fact that the equations of state $(\overline{108})$ and (113) are identical is a very nontrivial property of the effective Lagrangian (42). It is intimately related to the symmetries (global and local) which determine the form of this Lagrangian.

This calculation also shows that the diquarks Bose-condense [33], and thus form a superfluid. Since the diquarks are charged we can call this phase a superconducting phase.

\section{Conclusions and discussion}

In this paper we have derived the low-energy effective Lagrangian for certain QCD-like theories at finite chemical potential $\mu$. It contains the same number of phenomenological parameters as at $\mu=0$ : the pion decay constant $F$ and the vacuum value of the chiral condensate $\langle\bar{\psi} \psi\rangle_{0}$. Using this effective Lagrangian we have completely determined its lowenergy properties. In particular, we have established the dependence of the ground state, the condensates, and the masses of the excitations on the chemical potential $\mu$, the bare quark mass $m$ and the diquark source $j$. The theories to which our analysis applies include: (i) twocolor QCD with quarks in fundamental color representation, and (ii) any-color QCD with quarks in adjoint color representation. The unifying feature of these theories is that the lowenergy excitations, i.e. the Goldstone bosons of spontaneously broken symmetries, include baryons in the form of diquark states. Since the chiral Lagrangian describes the dynamics of such low-energy excitations, we can use the effective theory to describe phenomena associated with the condensation of diquarks.

Since we are working only to the lowest order in Chiral Perturbation Theory, we must remember that our approach has a limited region of applicability. First of all, the momenta of the particles, described by the Lagrangian (42) must be smaller than the scale $M$ of the masses of the non-Goldstone excitations, such as vector mesons, for example. In other words, the expansion parameter of the chiral perturbation theory is $p / M$. The bare quark mass

is also included to leading order in $m$. It is obvious from (42) that $m \sim m_{\pi}^{2} \sim p^{2}$ in the momentum power counting. The chemical potential $\mu$ is an external parameter with the dimension of mass. The interesting behavior of our theory is at values of $\mu \sim m_{\pi} \sim p$, and $\mu$ is counted as order $p$ in the momentum power counting. Our effective theory thus applies as long as $m_{\pi}$ and $\mu$ are much smaller than $M$.

Our effective theory predicts a phase transition at a value of $\mu=m_{\pi} / 2$. It is important to note that this transition occurs within the domain of applicability of the effective theory, as long as $m_{\pi}$ is small, as discussed above, unlike, for example, the transition in QCD with 
three colors. The transition at $\mu=m_{\pi} / 2$ is a robust prediction of our effective theory. The existence of such a transition is confirmed by lattice Monte Carlo simulations [ [ [ , 5].

The fact that our effective Lagrangian (42) predicts the transition at a value of $\mu$ equal to $m_{\pi} / 2$ is a direct consequence of the symmetries of the theory. The coefficient of the $\mu$-dependent term in the effective Lagrangian, responsible for the transition, is not an independent phenomenological parameter, but is fixed rigidly by the local symmetry [8]. This symmetry corresponds to the conservation of the baryon charge (among other generators of $\left.S U\left(2 N_{f}\right)\right)$. This ensures that the charge of a composite state in the effective theory is an additive sum of the charges of the constituents (non-renormalization). Indeed, the factor two in the relation $\mu_{0}=m_{\pi} / 2$ is the baryon charge of the diquark. This is in agreement with the value of $\mu$, below which no transition can occur at zero temperature, given by the minimum value of the mass per baryon number among all baryons in the theory (see, e.g., [32]). In three-color QCD this would correspond to a value of $\mu_{0}$ approximately equal to $1 / 3$ of the nucleon mass (offset by the binding energy of nuclear matter). [2 $^{2}$

Another way of looking at the $\mu$-dependence in the theory is the following. In the microscopic theory, given by the Lagrangian (36), $\mu$ enters as (a timelike component of) an Abelian gauge potential. As such, it can be completely removed from the Lagrangian by the time-dependent gauge transformation

$$
\psi \rightarrow e^{\mu \tau} \psi \quad \text { and } \quad \psi^{*} \rightarrow e^{-\mu \tau} \psi^{*},
$$

where $\tau$ is the Euclidean time ${ }^{[3}$. However, this does not mean that the partition function does not depend on $\mu$. Let us consider first the case of finite temperature. In this case, the boundary conditions in the Euclidean time direction for the quarks change after (114): they are no longer antiperiodic. The dependence on $\mu$ in the partition function comes entirely from the boundary conditions on the fermion fields

$$
\left.\psi\right|_{\tau=1 / T}=-\left.e^{\mu / T} \psi\right|_{\tau=0}, \quad \text { and }\left.\quad \psi^{*}\right|_{\tau=1 / T}=-\left.e^{-\mu / T} \psi^{*}\right|_{\tau=0}
$$

The $\mu$-independent Lagrangian with such boundary conditions is completely equivalent to the Lagrangian (36) and usual antiperiodic boundary conditions. This fact can be conveniently used in lattice simulations. The corresponding effective theory can also be defined with all $\mu$-dependence in the boundary conditions. The boundary conditions for a given effective (composite) bosonic field $\phi$ should then read $\phi(1 / T)=\exp (b \mu / T) \phi(0)$, where $b$ is the baryon charge of the field $\phi$ [34]. In the case of the matrix valued field $\Sigma$ we have

$$
\left.\Sigma\right|_{\tau=1 / T}=\left.\exp \left(\frac{\mu}{T} B\right) \Sigma\right|_{\tau=0} \exp \left(\frac{\mu}{T} B^{T}\right)
$$

where $B$ is the baryon charge matrix (35).

In the limit of zero temperature, our intuition suggests that the dependence of the partition function on boundary conditions should weaken and disappear. This is, however, not completely true for the boundary conditions such as (115) due to their singular nature in the limit $1 / T \rightarrow \infty$. There is indeed an interval of $\mu:-\mu_{0}<\mu<\mu_{0}$, where the partition

\footnotetext{
${ }^{12}$ We remind the reader that we measure the baryon charge in units of the $U(1)_{B}$ charge of a single quark.

13 Note that, in Euclidean formulation, the $\psi$ and $\psi^{*}$ are independent variables and the global flavor symmetry group is in fact $G l\left(2 N_{f}\right)$ [28.
} 
function does not depend on $\mu$. However, outside this interval, the singular nature of the boundary conditions starts playing a role: a phase transition occurs and a $\mu$-dependence appears.

Why is the approach of this paper not directly applicable to real three-color QCD? The main reason is that at $\mu=0$ the effective theory described by the chiral Lagrangian does not contain excitations with non-zero baryon number (pions do not carry baryon charge). It is easy to see that, as a consequence, applying either the method of local symmetry of Section 6.2 or the gauge transformation described in this section, one finds no dependence on $\mu$ in the effective chiral Lagrangian of QCD. Related to that is the fact that the value of $\mu_{0} \approx m_{\text {nucleon }} / 3$ is large, and the approach of this paper, based on an expansion in small $\mu$, will not reach it.

As we emphasized, the theories we considered are related by the fact that the fermion representations are pseudo-real. This is a consequence of the antiunitary symmetries of the Dirac operator (四), (2). We identified two such symmetry classes, distinguished by the Dyson index $\beta=1$ and $\beta=4$. The effective theories for these two classes of theories are very similar and dual, or complementary, to each other, from the point of view of the residual global symmetries. In the diquark (finite density) phase, for the $\beta=1$ theories the residual flavor symmetry is given by $S p\left(N_{f}\right)$, while for the $\beta=4$ case it is $S O\left(N_{f}\right)$. The excitations form multiplets which correspond to symmetric or antisymmetric second rank tensor representations of these groups (see Figs 1,2 and Tables 1,2). One can see that the $\beta=1$ and $\beta=4$ cases "mirror" each other with respect to $S p\left(N_{f}\right) \leftrightarrow S O\left(N_{f}\right)$ and symmetric $\leftrightarrow$ antisymmetric. On the other hand, the Dirac operator for three-color QCD with fundamental quarks does not have any antiunitary symmetries, and the fermion representations are complex. This case falls into the third remaining Dyson class with the index $\beta=2$.

It would be interesting to apply the approach of this paper to lattice theories with pseudoreal fermions, for example to two-color QCD with fundamental quarks. The symmetries of such theories are different from their continuum counterparts and were analyzed in [5]. In particular, the transition to continuum limit may turn our to be nontrivial. This can be related to the antiunitary symmetry of the lattice Dirac operator for staggered fermions in fundamental representation

$$
\mathcal{D}_{x y}=\frac{1}{2} \sum_{\mu} \eta_{x, \mu}\left(U_{x, \mu} \delta_{x+\hat{\mu}, y}-U_{x-\mu, \mu}^{\dagger} \delta_{x-\hat{\mu}, y}\right),
$$

where $\eta_{x, \mu}=(-1)^{x_{1}+\ldots+x_{\mu-1}}$, and $U$ are $S U(2)$ color matrices. The antiunitary symmetry of this lattice Dirac operator is given by $\tau_{2} \mathcal{D}=\mathcal{D}^{*} \tau_{2}$, or $\left[\mathcal{D}, \tau_{2} K\right]=0$. Since $\left(\tau_{2} K\right)^{2}=-1$, we conclude that such Dirac operator belongs to the class $\beta=4$. However, in the continuum limit, the Dirac operator must be in the class $\beta=1$. Such an observation was also made in [35, 36, 22, 5] from the point of view of global symmetries and their breaking, where it was pointed out that it is not yet known how the apparent pattern of $S U\left(2 N_{f}\right) \rightarrow O\left(2 N_{f}\right)$ breaking becomes $S U\left(2 N_{f}\right) \rightarrow S p\left(2 N_{f}\right)$ in the continuum. Even though the symmetry may modify many of the details of our analysis, when it is applied to lattice theories, some features should be robust. Such would include the phase transition at $\mu=m_{\pi} / 2$, the relation similar to $\langle\bar{\psi} \psi\rangle^{2}+\langle\psi \psi\rangle^{2}=$ const between the chiral and the diquark condensates, the linear 
dependence of $n_{B}$ on $\mu$ near the transition, the existence of several branches in the spectrum, similar to Figures 1, 2, 3, and many other qualitative features.

\section{Acknowledgements}

S. Hands, H. Leutwyler, M.-P. Lombardo, S. Morrison, E. Shuryak and D.K. Sinclair are acknowledged for useful discussions. J.B.K. is supported in part by the National Science Foundation, NSF-PHY96-05199. D.T. and J.J.M.V. are partially supported by the US DOE grant DE-FG-88ER40388. D.T. is supported in part by "Holderbank"-Stiftung and by Janggen-Pöhn-Stiftung. A.Z. is supported, in part, by the National Science and Engineering Research Council of Canada (NSERC).

\section{Appendix}

In this appendix we analyze the representations of the remaining symmetry groups shown in Table 1 for $\beta=1$. They are all different subgroups of $\operatorname{Sp}\left(2 N_{f}\right)$.

Under the symmetry group $S p\left(2 N_{f}\right)$ the Goldstone fields $\Sigma$ transform as

$$
\Sigma \rightarrow V \Sigma V^{T}
$$

With $\Sigma$ parameterized as $U \Sigma_{c} U^{T}$ (with $U \in S U\left(2 N_{f}\right)$ ) and $V \Sigma_{c} V^{T}=\Sigma_{c}$ (the $2 N_{f} \times$ $2 N_{f}$ antisymmetric unit matrix is denoted by $\Sigma_{c}$ ) it follows that the generators transform according to

$$
X \Sigma_{c} \rightarrow V X V^{-1} \Sigma_{c}=V X \Sigma_{c} V^{T} \quad \text { or } \quad\left(X \Sigma_{c}\right)_{i j} \rightarrow V_{i k} V_{j l}\left(X \Sigma_{c}\right)_{k l} .
$$

From the transposition relation (12) it follows that $\left(X \Sigma_{c}\right)^{T}=-X \Sigma_{c}$. If the symmetry group is $S p\left(2 N_{f}\right)$ the generators transform according to an antisymmetric rank two representation of $S p\left(2 N_{f}\right)$. The degeneracy is thus given by $2 N_{f}^{2}-N_{f}-1$. If the symmetry group is $S U\left(N_{f}\right) \times U(1)$ the symmetry transformation is given by

$$
V=\left(\begin{array}{cc}
U_{1} & 0 \\
0 & U_{1}^{*}
\end{array}\right),
$$

the $P$-type generators transform according to $P^{T} \rightarrow U_{1} P^{T} U_{1}^{-1}$ and the $Q$-type generators as $Q \rightarrow U_{1} Q U_{1}^{T}$. Since the $Q$ are antisymmetric they transform according to an antisymmetric rank two representation of $S U\left(N_{f}\right)$. The fields $Q^{\dagger}$ transform according to the conjugate representation. The dimension of both representations is equal to $N_{f}\left(N_{f}-1\right) / 2$. Since the $P$-type generators are traceless, the degeneracy is given by $N_{f}^{2}-1$.

In the diquark condensation phase the symmetry group $S p\left(2 N_{f}\right)$ is with respect to the rotated antisymmetric unit matrix, $\Sigma_{\alpha}$, with symplectic transformations defined by

$$
V \Sigma_{\alpha} V^{T}=\Sigma_{\alpha}
$$


According to the argument at the beginning of this appendix, the transformation properties of the generators are given by

$$
\left(X_{\alpha} \Sigma_{\alpha}\right)_{i j} \rightarrow V_{i k} V_{j l}\left(X_{\alpha} \Sigma_{\alpha}\right)_{k l}
$$

where the $X_{\alpha}$ are the rotated generators defined by

$$
X_{\alpha}=V_{\alpha} X V_{\alpha}^{-1}
$$

and $V_{\alpha}=\exp i \alpha X_{2} / 2$ defined in (59).

With $S p\left(N_{f}\right)$ as symmetry group the symmetry transformations are given by (120) with $U_{1}^{*}=-I U_{1} I$ (the $N_{f} \times N_{f}$ antisymmetric unit matrix is denoted by $I$ ). Since in this case $\left\{V, X_{2}\right\}=0$ and using that $\Sigma=V_{\alpha} U \Sigma_{c} U^{T} V_{\alpha}^{T}$ (see eq. (62)) we find that the representations can be discussed in terms of the fields $U \Sigma_{c} U^{T}$ with the familiar $P Q$-block structure of the generators. The $P$-type generators thus transform as $P^{T} I \rightarrow U_{1} P^{T} I U_{1}^{T}$. The symmetric and and antisymmetric components of $P I$ corresponding to $P_{S} I$ and $P_{A} I$, respectively, transform independently. The dimensions of the representations are given by $N_{f}\left(N_{f}+1\right) / 2$ and $N_{f}\left(N_{f}-\right.$ $1)-1$, respectively. The generators $Q$ and $I Q^{\dagger} I$ transform in the same way, and thus the linear combinations $\tilde{Q}$ and $\tilde{Q}^{\dagger}$ transform in the same way. Since $Q$ is antisymmetric they transform according to an irreducible rank two representation with dimension equal to $N_{f}\left(N_{f}-1\right) / 2$.

Finally, for symmetry group $S p\left(N_{f}\right) \times S p\left(N_{f}\right)$ the symmetry transformation is given by

$$
V=\left(\begin{array}{cc}
U_{1} & 0 \\
0 & U_{2}
\end{array}\right)
$$

with both $U_{1}$ and $U_{2}$ symplectic transformations. In this case it is imperative to consider the rotated generators. However, we only need the rotated generators for $\mu \rightarrow \infty$. In terms of the block structure of the unrotated generators, we obtain after a straightforward calculation for $\alpha=\pi / 2$,

$$
X_{\alpha} \Sigma_{\alpha}=\left(\begin{array}{cc}
-I P_{A} I+i Q_{I} I & i I P_{S}+Q_{R} \\
i P_{S} I+I Q_{R} I & P_{A}-i I Q_{I}
\end{array}\right) .
$$

Therefore the combination $i I P_{S}+Q_{R}$ transforms as

$$
i I P_{S}+Q_{R} \rightarrow U_{1}\left(i I P_{S}+Q_{R}\right) U_{2}^{T} .
$$

Indeed, this transformation corresponds to the Young tableaux given in upper right corner of Fig. 1. Notice that for $\mu \rightarrow \infty$ we have that $\tilde{Q}^{\dagger} \rightarrow Q_{R}$. The 11- and 22-blocks of the matrix (125) correspond to two different linear combinations of $P_{A}$ and $Q_{I}$. One combination transforms as a rank two tensor with respect to $U_{1}$, and the other combination as a rank two tensor with respect to $U_{2}$. Since both combinations are antisymmetric with respect to transposition, they transform according to the Young tableaux given in the lower right corner of Fig. 1. The additional singlet terms arise because the irreducible representations are traceless.

In case of $S p\left(N_{f}\right)$ symmetry, $U_{2}=U_{1}^{*}=-I U_{1} I$, the transformation properties of $P_{S}$, $P_{A}, Q_{R}$ and $Q_{I}$ can be obtained from (125) by combining the transformation properties of 
the diagonal blocks and of the off-diagonal blocks. The results are in agreement with the discussion in the paragraph following eq. (123).

The same analysis can be performed for $\beta=4$. In fact, because the matrix $I$ is absent, this case is somewhat simpler, and we leave it as an exercise to the reader.

\section{References}

[1] See e.g. Proceedings of QCD at Finite Baryon Density, Bielefeld, April 1998, F. Karsch and M.-P Lombardo, eds, Nucl. Phys. A 642, 1998.

[2] J.B. Kogut, M.P. Lombardo and D.K. Sinclair, Phys. Rev. D 51 (1995) 1282; Nucl. Phys. B, Proc. Suppl. 42 (1995) 514; I.M. Barbour, S.E. Morrison, E.G. Klepfish, J.B. Kogut, M.P. Lombardo, Nucl. Phys. Proc. Suppl. 60A (1998) 220.

[3] D. Bailin and A. Love, Phys. Rept. 107 (1984) 325; M. Alford, K. Rajagopal and F. Wilczek, Phys. Lett. B422 (1998) 247, Nucl. Phys. B537 (1999) 443; R. Rapp, T. Schäfer, E.V. Shuryak and M. Velkovsky, Phys. Rev. Lett. 811998 53; Ann. Phys. 280 (2000) 35.

[4] E. Dagotto, F. Karsch, and A. Moreo, Phys. Lett. B 169 (1986) 421; E. Dagotto, A. Moreo, and U. Wolff, Phys. Rev. Lett. 57 (1986) 1292; Phys. Lett. B 186 (1987) 395.

[5] S. Hands, J.B. Kogut, M.-P. Lombardo, S.E. Morrison, Nucl. Phys. B 558 (1999) 327; S. Hands and S.E. Morrison, hep-lat/9902012, hep-lat/9905021.

[6] T. Schäfer, Phys. Rev. D57 (1998) 3950.

[7] M.A. Stephanov, Phys. Rev. Lett. 76 (1996) 4472; Nucl. Phys. Proc. Suppl. 53 (1997) 469.

[8] J. Kogut, M.A. Stephanov and D. Toublan, Phys. Lett. B464 (1999) 183.

[9] R.F. Alvarez-Estrada and A. Gomez Nicola, Phys.Lett. B355 (1995) 288; errat. Phys.Lett. B380 (1996) 491.

[10] C. DeTar, Quark-gluon plasma in numerical simulations of QCD, in Quark gluon plasma 2, R. Hwa ed., World Scientific 1995.

[11] A.V. Smilga, Phys. Rep. 291, (1997) 1.

[12] W. Pauli, Nuovo Cimento 6 (1957) 205 ; F. Gürsey, Nuovo Cimento 7 (1958) 411.

[13] S. Duane and J.B. Kogut, Nucl. Phys. B275 (1986) 398.

[14] S. Duane, A.D. Kennedy, B.J. Pendleton and D. Roweth, Phys. Lett. B195 (1987) 216.

[15] V.A. Novikov, M.A. Shifman, A.I. Vainshtein and V.I. Zakharov, Nucl. Phys. B260 (1985) 157. 
[16] C. Vafa and E. Witten, Nucl. Phys. B234 (1984) 173.

[17] S. Dimopoulos, Nucl. Phys. B168 (1980) 69; M. Vysotskii, Y. Kogan and M. Shifman, Sov. J. Nucl. Phys. 42 (1985) 318; D.I. Diakonov and V.Yu. Petrov, Lecture notes in physics, 417, Springer 1993.

[18] M.E. Peskin, Nucl. Phys. B175 (1980) 197.

[19] A. Smilga and J.J.M. Verbaarschot, Phys. Rev. D51 (1995) 829.

[20] F.J. Dyson, J. Math. Phys. 3 (1962c) 1199.

[21] E.V. Shuryak and J.J.M. Verbaarschot, Nucl. Phys. A560 (1993) 306.

[22] J.J.M. Verbaarschot, Phys. Rev. Lett. 72 (1994) 2531; Phys. Lett. B329 (1994) 351.

[23] M. Zirnbauer, J. Math. Phys. 37 (1996) 4986.

[24] J.J.M. Verbaarschot, Lectures given at APCTP - RCNP Joint International School on Physics of Hadrons and QCD, Osaka, Japan, 1998 and the 1998 YITP Workshop on QCD and Hadron Physics, Kyoto, Japan, 1998, hep-ph/9902394.

[25] M.A. Halasz, J.C. Osborn and J.J.M. Verbaarschot, Phys. Rev. D56 (1997) 7059.

[26] S. Weinberg, Physica A96 (1979) 327.

[27] J. Gasser and H. Leutwyler, Ann. Phys. 158, 142 (1984); J. Gasser and H. Leutwyler, Nucl. Phys. B250, 465 (1985); H. Leutwyler, Ann. Phys. 235 (1994) 165.

[28] D. Toublan and J.J.M. Verbaarschot, Nucl. Phys. B560 (1999) 259.

[29] Y.V. Fyodorov, B.A. Khoruzenko and H.-J. Sommers, Phys. Lett. A226 (1997) 46; Y.V. Fyodorov, M. Titov and H.-J. Sommers, Phys. Rev. E58 (1998) 1195.

[30] K.B. Efetov, Phys. Rev. Lett. 79 (1997) 491; Phys. Rev. B56 (1996) 9630; A.V. Kolesnikov and K.B. Efetov, Waves in Random Media 9 (1999) 71.

[31] L.D. Landau, E.M. Lifshitz and L.P. Pitaevskii, Statistical Physics, Part 2, v. 9, Ch.25; Oxford ; New York : Pergamon Press, 1980.

[32] M.A. Halasz, A.D. Jackson, R.E. Shrock, M.A. Stephanov and J.J.M. Verbaarschot, Phys. Rev. D58 (1998) 096007.

[33] E. Shuryak, Lectures given at NATO Advanced Study Institute, in "Confinment, Duality and Nonperturbative Aspects of QCD", Cambridge, 1997, 307.

[34] T.S. Evans, hep-ph/9510298.

[35] J.B. Kogut, H. Matsuoka, M. Stone, H.W. Wyld, S. Shenker, J. Shigemitsu and D.K. Sinclair, Nucl. Phys. B225 (1983) 93.

[36] S. Hands and M. Teper, Nucl. Phys. B347 (1990) 819. 